2

3

4

5

6

8

25

26 7

\title{
METABOLIC FINGERPRINT OF GESTATIONAL DIABETES MELLITUS
}

\author{
Danuta Dudzik ${ }^{1,2}$, Marcin Zorawski², Mariusz Skotnicki ${ }^{3}$, Wieslaw Zarzycki ${ }^{4}$, Gabryela \\ Kozlowska ${ }^{4}$, Katarzyna Bibik-Malinowska ${ }^{3}$, María Vallejo ${ }^{1}$, Antonia García ${ }^{1}$, Coral Barbas ${ }^{1}$, \\ M.Pilar Ramos 5
}

${ }^{1}$ CEMBIO (Center for Metabolomics and Bioanalysis), Facultad de Farmacia, Universidad CEU

8 San Pablo, Madrid, Spain; ${ }^{2}$ Department of Pharmacology, Medical University of Bialystok, 9 Bialystok, Poland; ${ }^{3}$ Clinical Department of Perinatology, Public Clinic Hospital, Medical University of Bialystok, Bialystok, Poland; ${ }^{4}$ Clinical Department of Endocrinology, Diabetology 1 and Internal Diseases, Public Clinic Hospital, Medical University of Bialystok, Bialystok, 2 Poland; ${ }^{5}$ Biochemistry and Molecular Biology, Facultad de Farmacia, Universidad CEU San Pablo, Madrid, Spain. 4

5 Running title: Metabolic fingerprint of Gestational Diabetes Mellitus

Keywords: Gestational Diabetes Mellitus; Maternal Metabolism; Metabolic fingerprinting

To whom correspondence should be adressed:

$\mathrm{M}^{\mathrm{a}}$ del Pilar Ramos Álvarez, PhD.

Facultad de Farmacia

Universidad San Pablo CEU

Ctra. Boadilla del Monte km 5,3

28668, Madrid

+34-91-3724760

4 pramos@ceu.es 
29 Gestational Diabetes (GDM) is causing severe short- and long-term complications for mother,

30 fetus or neonate. As yet, the metabolic alterations that are specific for the development of GDM

31 have not been fully determined, which also precludes the early diagnosis and prognosis of this

32 pathology. In this pilot study, we determine the metabolic fingerprint, using a multiplatform LC-

33 QTOF/MS, GC-Q/MS and CE-TOF/MS system, of plasma and urine samples of 20 women with

34 GDM and 20 with normal glucose tolerance in the second trimester of pregnancy. Plasma

35 fingerprints allowed for the discrimination of GDM pregnant women from controls. In particular,

36 lysoglycerophospholipids showed a close association with the glycemic state of the women. In

37 addition, we identified some metabolites with a strong discriminative power, such as LPE(20:1),

38 (20:2), (22:4); LPC(18:2), (20:4), (20:5); LPI(18:2), (20:4); LPS(20:0) and LPA(18:2), as well as

39 taurine-bile acids and long-chain polyunsaturated fatty acids derivatives. Finally, we provide

40 evidence for the implication of these compounds in metabolic routes, indicative of low-grade

41 inflammation and altered redox-balance, that may be related with the specific pathophysiological

42 context of the genesis of GDM. This highlights their potential use as prognostic markers for the

43 identification of women at risk to develop severe glucose intolerance during pregnancy.

Biological Significance:

46 Gestational Diabetes Mellitus (GDM) is increasing worldwide and, although diabetes usually

47 remits after pregnancy, women with GDM have a high risk of developing postpartum type 2-

48 diabetes, particularly when accompanied by obesity. Therefore, understanding the 
49 pathophysiology of GDM, as well as the identification of potentially modifiable risk factors and 50 early diagnostic markers for GDM are relevant issues. In the present study, we devised a 51 multiplatform metabolic fingerprinting approach to obtain a comprehensive picture of the early

52 metabolic alternations that occur in GDM, and may reflect on the specific pathophysiological 53 context of the disease. Future studies at later stages of gestation will allow us to validate the 54 discriminant power of the identified metabolites.

55 
56 Gestational Diabetes Mellitus (GDM), defined as "any degree of glucose intolerance with onset

57 or first recognition during pregnancy" [1], is increasing worldwide and, depending on the

58 population analyzed and on the diagnostic criteria used, its prevalence ranges from $3 \%-14 \%$ of

59 all pregnancies. Despite advances in diagnosis and good maternal control [2], GDM is frequently

60 causing short- and long-term health complications for the mother, the fetus and the neonate [3].

61 Furthermore, although diabetes usually remits after pregnancy, women with GDM have a high

62 risk of developing postpartum type 2-diabetes, particularly when accompanied by obesity [4].

63 There is lack of international uniformity regarding the ascertainment and diagnosis of GDM.

64 Therefore, understanding the pathophysiology of GDM, as well as the identification of

65 potentially modifiable risk factors and early diagnostic markers for GDM are relevant issues.

66 Contemporary "omics" approaches, in particular metabolomics, provide deeper insights in the

67 etiopathogenesis and discovery of biomarkers of diseases. A unique and disease-specific

68 metabolite pattern or "fingerprint" allows for deciphering biological processes, and for the

69 identification of compounds with potential diagnostic or predictive power. A growing number of

70 metabolomics studies aimed at uncovering the metabolic signature of type 2-diabetes [5, 6],

71 focusing on potential biomarkers of altered glucose tolerance and onset of insulin resistance,

72 such as branched-chain amino acids, acylcarnitines, choline-containing phospholipids and 2-

73 hydroxybutyrate [7].

74 In the present study, we devised a multiplatform metabolic fingerprinting approach to obtain a 75 comprehensive picture of the early metabolic alternations that occur in GDM, and to eventually 76 identify potential biomarkers that predict the risk of the GDM pregnant women to develop severe 
77 glucose intolerance that will be validated in future studies at later stages of gestation and on an 78 independent cohort of women.

RESEARCH DESIGN AND METHODS

\section{Study population}

82 GDM screening was done routinely at 22-28 weeks of gestation after overnight fasting by an oral

83 glucose tolerance test (OGTT). According to WHO-1998 criteria, GDM was defined as glucose

84 level $\geq 140 \mathrm{mg} / \mathrm{dl}(7.8 \mathrm{mmol} / \mathrm{l})$ after 2 -h 75 -g OGTT. Women known to have previous diabetes

85 mellitus or other complications were excluded from the study. Finally, twenty caucasian women

86 with GDM and 20 healthy caucasian pregnant women with normal glucose tolerance were

87 matched according to week of gestation and age (22-37 years). At the day of the OGTT, venous

88 fasting blood samples were drawn into EDTA-containing tubes and overnight urine was

89 collected. Samples were stored at $-80^{\circ} \mathrm{C}$ until analysis. The study was carried out in accordance

90 with the permission of the Bioethical Commission of the Medical University of Bialystok,

91 Poland. Written informed consent was obtained from each participant in the study.

\section{Biochemical analysis and indices of insulin resistance}

94 Plasma glucose, cholesterol, LDL/HDL-cholesterol, triacylglycerols and C-reactive protein 95 (CRP) were measured in an autoanalyzer (Cobas C111 Roche Autoanalyzer, Hoffmann-LaRoche 96 Ltd., Basel Switzerland). Blood HbA1c was analyzed by the D-10 ${ }^{\mathrm{TM}}$ Hemoglobin Testing 97 System (Bio-Rad, USA), C-peptide by an ELISA kit (Biosource International, Inc., Belgium), 
and insulin with an INS-IRMA-RIA kit (DIAsource ImmunoAssays S.A., Belgium). HOMA-IR (Homeostatic Model Assessment) [8] and QUICKI (Quantitative Insulin Sensitivity Check Index) [9] indices were calculated with fasting glucose $(\mathrm{mg} / \mathrm{dL})$ and insulin $(\mu \mathrm{U} / \mathrm{mL})$ as described. The area under the curve (AUC-G) for glucose during the OGTT was determined by the trapezoidal method with Prism 6.0 software.

\section{Metabolic fingerprinting}

Standards for GC-MS and organic solvents were from Sigma-Aldrich (Madrid, Spain); standards and reference mass solutions for LC-MS and CE-MS were from Agilent Technologies (Madrid, Spain).

Sample preparation was done according to standard protocols [10-12]. Briefly, for LC-MS analysis, proteins were precipitated by mixing 1 volume of plasma with 3 volumes of methanol/ethanol (1:1); for GC-MS analysis, protein precipitation was performed by treatment with cold acetonitrile (1:3), followed by methoximation with $O$-methoxyamine hydrochloride $(15 \mathrm{mg} / \mathrm{mL})$ in pyridine, and silylation with $\mathrm{N}, \mathrm{O}-$ bis(trimethylsilyl)trifluoroacetamide in $1 \%$ trimethylchlorosilane. Finally, urine samples for CE-MS analysis were prepared by incubating 1 volume of urine with 4 volumes of $0.125 \mathrm{M}$ formic acid. Quality control (QC) samples were prepared by pooling equal volumes of each sample and were injected every 6 samples injections and at the beginning/end of each analysis [13].

Fingerprinting of plasma with LC-QTOF/MS. A UHPLC system (Agilent 1290 Infinity LC System), equipped with a degasser, two binary pumps, and a thermostated autosampler coupled with Q-TOF LC/MS (6550 iFunnel) system (Agilent), was used in the ESI+ and ESI- mode to increase the number of detected metabolite ions as we previously described [10]. Briefly, $0.5 \mu \mathrm{L}$ 
122 of extracted plasma samples were injected into a thermostated $\left(60^{\circ} \mathrm{C}\right) \mathrm{RP}$ Zorbax Extend $\mathrm{C}_{18}$ 123 column $(2.1 \times 50 \mathrm{~mm}, 1.8 \mu \mathrm{m}$; Agilent Technologies $)$. The flow rate was $0.6 \mathrm{~mL} / \mathrm{min}$ with 124 solvent A (water with $0.1 \%$ formic acid), and solvent B (acetonitrile with $0.1 \%$ formic acid). The 125 chromatographic gradient started at 5\% phase B during the first minute, followed by and increase 126 of phase B to $80 \%$ (1-7 $\mathrm{min}$ ) and $100 \%$ (7-11.5 $\mathrm{min})$; the system was re-equilibrated by reverting

127 the gradient to $5 \%$ phase B (12-15 min). The system was operated in full scan mode from 50$1281000 \mathrm{~m} / \mathrm{z}$ for positive and $50-1100 \mathrm{~m} / \mathrm{z}$ for negative mode. Capillary voltage was set to $3 \mathrm{kV}$ for 129 positive and negative ionization modes; the drying gas flow rate was $12 \mathrm{~L} / \mathrm{min}$ at $250{ }^{\circ} \mathrm{C}$ and gas 130 nebulizer at $52 \mathrm{psi}$; fragmentor voltage was $175 \mathrm{~V}$ for positive and $250 \mathrm{~V}$ for negative ionization 131 mode; skimmer and octopole radio frequency voltage (OCT RF Vpp) were set to 65V and 750V, 132 respectively. Data were collected in the centroid mode at a scan rate of 1.0 spectrum per second. 133 Accurate mass measurements were obtained by means of an automated Calibrant Delivery 134 System (CDS), using a Dual Agilent Jet Stream Electrospray Ionization (Dual AJS ESI) source 135 that continuously introduces a calibrant solution with reference masses at $\mathrm{m} / \mathrm{z} 121.0509$ $136\left(\mathrm{C}_{5} \mathrm{H}_{4} \mathrm{~N}_{4}\right)$ and $\mathrm{m} / \mathrm{z} 922.0098\left(\mathrm{C}_{18} \mathrm{H}_{18} \mathrm{O}_{6} \mathrm{~N}_{3} \mathrm{P}_{3} \mathrm{~F}_{24}\right)$ in positive ionization mode or $\mathrm{m} / \mathrm{z} 112.98568$ $137\left(\mathrm{C}_{2} \mathrm{O}_{2} \mathrm{~F}_{3}\left(\mathrm{NH}_{4}\right)\right)$ and $1033.9881\left(\mathrm{C}_{18} \mathrm{H}_{18} \mathrm{O}_{6} \mathrm{~N}_{3} \mathrm{P}_{3} \mathrm{~F}_{24}\right)$ in negative ionization mode. Samples were 138 analyzed in separate runs (positive and negative ionization mode), in a randomized order.

140 Fingerprinting of plasma with GC-Q-MS. A GC system (Agilent Technologies 7890A), 141 equipped with an autosampler (Agilent 7693) and interfaced to an inert mass spectrometer with 142 triple-Axis detector (5975C, Agilent), was used for fingerprinting as we have previously 143 described $[11,14]$. Briefly, $2 \mu \mathrm{L}$ of the derivatized sample were injected in a GC-Column DB5- 
144 MS (30 m length, $0.25 \mathrm{~mm}, 0.25 \mu \mathrm{m}$ film 95\% dimethyl/ 5\% diphenylpolysiloxane) couple to a 145 pre-column (10 m J\&W integrated with Agilent 122-5532G). The injector port was held at 250 $146{ }^{\circ} \mathrm{C}$, and the helium carrier gas flow rate was set at $1.0 \mathrm{~mL} / \mathrm{min}$. The split ratio was 1:10. The 147 temperature gradient was programmed as follows: the initial oven temperature was set to $60{ }^{\circ} \mathrm{C}$ 148 (held for $1 \mathrm{~min}$ ), increased to $325^{\circ} \mathrm{C}$ at a rate of $10^{\circ} \mathrm{C} / \mathrm{min}$; the system was allowed to cool down 149 for $10 \mathrm{~min}$ before the next injection. The detector transfer line, the filament source and the 150 quadrupole temperature were set to $280{ }^{\circ} \mathrm{C}, 230^{\circ} \mathrm{C}$ and $150{ }^{\circ} \mathrm{C}$, respectively. MS detection was 151 performed in electron impact (EI) mode at $-70 \mathrm{eV}$. The mass spectrometer was operated in scan 152 mode over a mass range of $50-600 \mathrm{~m} / \mathrm{z}$ at a rate of $2.7 \mathrm{scan} / \mathrm{s}$.

154 Fingerprinting of urine with $\boldsymbol{C E}-\mathbf{T O F} / \mathbf{M S}$. An Agilent 7100 (CE) system, coupled to a TOF 155 Mass Spectrometer (6224 Agilent), was used for samples analysis as published previously [12]. 156 In brief, a fused-silica capillary (Agilent Technologies; total length, $96 \mathrm{~cm}$; i.d., $50 \mu \mathrm{m}$ ) was pre157 conditioned with $1 \mathrm{M} \mathrm{NaOH}$ for 30 min, followed by MilliQ ${ }^{\circledR}$ water for 30 min and background 158 electrolyte - BGE (0.8 M formic acid in 10\% methanol) for $30 \mathrm{~min}$. Before each analysis, the 159 capillary was flushed for 5 min (950 mbar pressure) with BGE. The MS was operated in positive 160 polarity, with a full scan from 80 to $1000 \mathrm{~m} / \mathrm{z}$ at a rate of $1.4 \mathrm{scan} / \mathrm{s}$. Drying gas was set to 10 $161 \mathrm{~L} / \mathrm{min}$, nebulizer to $10 \mathrm{psi}$, voltage to $3.5 \mathrm{kV}$, fragmentor to $100 \mathrm{~V}$, gas temperature to $200{ }^{\circ} \mathrm{C}$ 162 and skimmer to $65 \mathrm{~V}$. The sheath liquid composition was methanol/water (1/1, v/v), containing $1631.0 \mathrm{mmol} / \mathrm{L}$ formic acid with two references masses $\left(121.0509\right.$ - purine $\left(\mathrm{C}_{5} \mathrm{H}_{4} \mathrm{~N}_{4}\right)$ and 922.0098 164 - HP-921 $\left.\left(\mathrm{C}_{18} \mathrm{H}_{18} \mathrm{O}_{6} \mathrm{~N}_{3} \mathrm{P}_{3} \mathrm{~F}_{24}\right)\right)$, which allows for correction and provides more accurate mass 165 determination. Flow rate was $0.6 \mathrm{~mL} / \mathrm{min}$ and split was set to 1/100. Samples were injected at 50 166 mbar for $17 \mathrm{~s}$. After each injection, along with the samples, BGE was co-injected for $10 \mathrm{~s}$ at 100 
167 mbar pressure to improve repeatability. Separations were performed at a pressure of 25 mbar and 168 a voltage of $+30 \mathrm{kV}$; current under these conditions was $25 \mu \mathrm{A}$.

\section{Data acquisition and statistical analysis}

171 To provide quality assurance of results, LC-MS, GC-MC, CE-MS data treatment was performed

172 as described previously [15]. Briefly, LC-MS and CE-MS raw data were cleaned from

173 background noises and unrelated ions by the Molecular Feature Extraction tool (MassHunter

174 Qualitative Analysis Software; Agilent). GC-MS data were analysed using the Agilent 175 ChemStation Software (G1701EA E.02.00.493, Agilent). AMDIS 2.69 software from NIST 176 (U.S. National Institute of Standards and Technology) was used for mass spectral deconvolution 177 to identify co-eluted compounds according to their retention indices and retention times. GC-MS 178 and CE-MS data were normalized according to C18:0 methyl ester and creatinine intensity, 179 respectively. Primary data treatment (filtering and alignment) was performed with MPP (Mass

180 Profiler Professional) B.12.1 software (Agilent). Variation of compound responses in QC181 samples was expressed as CV. Metabolites detected in $<50 \%$ of all QC-samples and with a CV $182>30 \%$ were removed to asure repeatability.

183 Univariate statistical analysis assuming equal ( $t$-test) or unequal variance (Welch's $t$-test) and 184 hierarchial heat map clustering analysis were performed with MPP B.12.1 software. Benjamin 185 Hochberg FDR was applied for $P$ value correction. Multivariate (unsupervised and supervised) 186 analysis was performed using SIMCA-P+ 12.0 (Umetrics, Umea, Sweden). Associations 187 between variables were tested by Spearman correlation coefficients $\left(\mathrm{r}_{s}\right)$ using Prism 6.0 software. 188 Assessment of the diagnostic performance of the metabolites was made using the receiver 
189 operating characteristic (ROC) curves by plotting the sensitivity against the corresponding false190 positive rate (100-specificity), with the Prism 6.0 software. A test with perfect discrimination 191 power yields a ROC curve that passes through the upper left corner with an AUC of one (100\% 192 sensitivity and $100 \%$ specificity). Thus, the closer the ROC-area to one, the higher the 193 discriminant power of the metabolite. To construct the ROC curves, GDM was defined 194 according to WHO-1998 criteria, as glucose level $\geq 140 \mathrm{mg} / \mathrm{dl}$ (7.8 mmol/l) after 2-h 75-g OGTT. 195 To establish potential cutoff values for each metabolite, we determined the optimal decision 196 point from the ROC curve, assigning equal weights to the sensitivity and specificity of the test.. 197 Then, best sensitivity, specificity and likelyhood ratio for the selected cut-off of each parameter 198 were obtained.

\section{Compound identification}

201 Identification of compounds (LC-MS and CE-MS) that were significant in class separation was 202 performed by searching accurate masses against online databases (METLIN, HMDB, KEGG, 203 LIPIDMAPS), and confirmed by LC-MS/MS. For CE-MS, compound identification was 204 confirmed by using available standards. Compound identification by GC-MS was performed 205 with the target metabolite Fiehn GC/MS Metabolomics RTL library (G1676AA, Agilent), the 206 CEMBIO-library and the NIST mass spectra library 2.0, using the ChemStation software and 207 native PBM (Probability-Based Matching) algorithm (G1701EA E.02.00.493, Agilent). 
210

211

212

213

\section{RESULTS}

\section{Study participants}

216 There was no difference in age, parity or blood pressure between the women in the different 217 groups (Table 1). BMI before gestation (BMIo) was similar in control and GDM women, despite 218 a significantly higher BMI in $2^{\text {nd }}$ trimester (BMI-2t). As expected, women who were classified as 219 GDM according to WHO-criteria, had significantly higher fasting glucose, HbA1c, 220 221

222

Models obtained by multivariate calculations were validated by a cross-validation tool [16], using the "leaving one third out" approach. Prediction of excluded samples was reiterated until all samples were predicted at least once.

GDM according to WHO-criteria, had significantly higher fasting glucose, HbAlc,
triacylglycerides and cholesterol than controls; HDL and LDL-cholesterol, basal insulin, Cpeptide and CRP levels did not differ. During the OGTT, glucose levels were significantly higher at one and 2 hours in the women classified as GDM. The AUC-G was higher in the GDM women than in controls; there were no significant differences in HOMA-IR and QUICKI.

Correlation analysis shows that there was no association between the body weight gain (BMI-2t minus BMIo) and various measures of glycemic control and insulin resistance. We observed that both, BMIo and BMI-2t, correlated with glycemic condition ( $\mathrm{r}_{s}$ for correlation with BMI-2t were $0.368,0.424,0.355,0.485$ and -0.455 for basal glucose, AUC-G, insulin, HOMA-IR and QUICKI, respectively; $P<0.05$ for all correlations). Significant correlations were also observed for plasma triacylglycerides and basal or 2h-glucose, AUC-G, insulin, HOMA-IR and QUICKI $\left(\mathrm{r}_{s}=0.412 ; 0.446 ; 0.460 ; 0.386 ; 0.453\right.$ and -0.501 , respectively, $P<0.01$ for all correlations $)$. No correlations were found between cholesterol (total, LDL, HDL) and basal or 2h-glucose, the 
AUC-G, HOMA-IR and QUICKI indices (data not shown).

\section{Metabolic fingerprinting}

235 Metabolic fingerprinting allowed for the simultaneous detection of 114,431 potential compounds 236 in plasma (LC: 114,347; GC: 84) and 7,428 in urine (CE). Data were filtered by choosing only 237 those present in 100\% (LC-MS/GC-MS) or 85\% (CE-MS) of the samples in at least one of the 238 groups. PCA analysis was used as an unsupervised method to get an overview and to detect 239 trends within these data (626 in ESI+, 487 in ESI-; 48 in GC-MS; 127 in CE-MS). For LC-MS 240 data, a clear separation can be observed between GDM and control groups, indicating metabolic 241 changes inherent to GDM (Fig. 1A-B).

242 Supervised Partial Least Squares Discriminant Analysis (PLS-DA) and Orthogonal PLS 243 Discriminant Analysis (OPLS-DA) were used for modeling differences between the groups. 244 Figure $1 C-F$ highlights the quality of the models, allowing for a clear separation of samples. To 245 estimate the predictive power of the analysis, a cross-validation of PLS-DA models was 246 performed. In the models obtained with data from LC-MS, 94\% (ESI+) and 100\% (ESI-) of all 247 excluded samples were classified correctly; in data from GC-MS and CE-MS, 79\% and 85\%, 248 respectively, of excluded samples were classified correctly.

249 Based on the compounds identified by LC-MS, we generated a metabolite heat map that revealed 250 considerable differences between control and diabetic women (Fig. 2). Based on these findings, 251 individual metabolites were compared, yielding statistical differences between control and GDM 252 women in 571 metabolites in plasma (558 by LC-MS; 13 by GC-MS) and in 72 in urine (CE- 
253 MS). To ensure valid measurements, metabolites with very high analytical variance (determined 254 as $\mathrm{CV}$ in QC) were excluded from further analysis. Finally, we identified 142 compounds in 255 plasma that were statistically different between the groups, including 83 glycerophospholipids 256 (51 lyso- and 32 phosphoglycerides), 9 sphingophospholipids $(6$ sphingomyelins, 2 257 sphingoethanolamines, sphingosine phosphate); 25 fatty acids or derivatives (3 fatty acids, 20 258 modified fatty acids, 1 eicosanoid, 1 ketone body); carnitine and 5 acylcarnitines; 7 amino acids 259 or modified aminoacids; 4 bile acid and derivatives; pyruvic and fumaric acid; glycerol; 1 260 vitamin; creatinine; 2-hydroxybutyrate, and 2 other compounds. In urine we identified 6 261 metabolites, including 5 aminoacids or derivatives, and carnitine. The most pronounced GDM262 specific changes corresponded to lysophosphoglycerides, being the most abundant compounds 263 lysophosphatidylcholines (LPC) with 16:0, 18:0, 18:1, 18:2, 18:3, 20:3, 20:4 and 20:5 acyl 264 chains, followed by lysophosphatidylethanolamines (LPE) with 16:0, 18:0, 18:2, 20:0, 20:1, $26520: 2,22: 4$ and 22:6 chains.

266 Table 2 shows metabolites that exhibited the highest significant differences between case and 267 control groups. A complete list of metabolites identified by LC-MS that differed between 268 experimental groups is available as supplementary material (Table 1S). Among the numerous 269 glycerophospholipids that were determined, $\operatorname{LPE}(20: 1), \operatorname{LPE}(20: 2), \operatorname{LPE}(22: 4), \operatorname{LPC}(20: 5)$, 270 LPC(18:2), LPC(18:1), LPI(20:4), LPS(20:0), lysophosdatidic acid LPA(18:2), lipoxin C4, and 271 the taurine-conjugates bile acids, trihydroxy-cholestanoyl taurine and taurolythocholic acid 272 glucuronide showed a pronounced decrease with gestational diabetes, followed by other 273 glycerophospholipids-species with poly-unsaturated fatty acids (PUFAs) as acyl moiety, 274 glycerophosphocoline, long-chain PUFA (LC-PUFA) derivatives, such as araquidonate or 275 docosahexaenoic acid methyl esters, conjugates bile acids, glycerophospholipids, 
276 sphingophospholipids and acylcarnitines. Some metabolites showed an increase in the GDM 277 group as compared to controls, including PE(38:6), PE(36:5), PC(38:1), PC(40:3), acetyl278 carnitine, linoleic acid, glycerol, 3-hydroxybutyrate, 2-hydroxybutyrate, and fumaric acid (Table 279 2). In urine, we found a significant decrease of carnitine in GDM pregnant women, whereas 280 hystidine, glutamine, phenylalanine, tryptophan and cystine were augmented in GDM.

281 We further compared the fatty acid chain of the lysoglycerophospholipids affected by GDM, and 282 found not only a decrease in the total amount of lysoglycerophospholipids, but also a 1.7 fold 283 increase of the ratio of saturated versus unsaturated fatty acids in GDM women as compared to 284 controls (Fig. 3).

285 We performed a correlation analysis between all metabolites and diabetes outcome measures 286 (fasting glucose, OGTT dynamics, HbA1c, HOMA-IR and QUICKI indices). Hundred and 287 thirty-five metabolites correlated with the $2 \mathrm{~h}$-glucose (92 with $P<0.001)$. Most metabolites 288 correlated also with AUC-G, or HbA1c, but not with HOMA-IR or QUICKI. For simplicity, only 289 those compounds that exhibited the highest Spearman coefficients $(>+/-0.65)$ with 2 -h glucose 290 are shown (Table 3); correlations with fasting glucose, AUC-G, HbA1c and HOMA-IR are also 291 included. Among metabolites that correlated with $2 \mathrm{~h}$-glucose, approximately $40 \%$ were 292 lysoglycerophospholipids with a LC-PUFA moiety. The strongest associations were observed 293 between 2h-glucose and arachidonic acid methylester $\left(\mathrm{r}_{s}=-0.7984\right)$, LPS (21:0) $\left(\mathrm{r}_{s}=0.7971\right)$, $294 \operatorname{LPE}(20: 1) \quad\left(\mathrm{r}_{s}=-0.7934\right)$, trihydroxy-cholanoyl taurine $\left(\mathrm{r}_{s}=-0.7893\right), \operatorname{LPE}(20: 2) \quad\left(\mathrm{r}_{s}=-0,7812\right)$, $295 \operatorname{LPC}(18: 2) \quad\left(\mathrm{r}_{s}=-0,7713\right), \quad \operatorname{LPC}(20: 4) \quad\left(\mathrm{r}_{s}=-0,7684\right), \quad \operatorname{LPC}(18: 1) \quad\left(\mathrm{r}_{s}=-0.7658\right), \quad \operatorname{LPI}(18: 2) \quad\left(\mathrm{r}_{s}=-\right.$ 296 0.7649), LPS (20:0) $\left(\mathrm{r}_{s}=-0.7633\right) ; \operatorname{LPI}(20: 4)\left(\mathrm{r}_{s}=-0.7576\right)$ and $\operatorname{LPC}(20: 5)\left(\mathrm{r}_{s}=-0.7531\right)$. Other 297 lysoglycerophospholipids and glycerophospholipids with PUFAs in their lipid moiety had also 298 significant strengths of association. Linoleic acid and dodecamide showed positive correlation 
299 with $2 \mathrm{~h}$-glucose $(P<0.05)$, whereas cerebronic acid and other fatty acid derivatives showed a 300 negative correlation with the various measures of the glycemic state.

301 Other metabolites that were altered in GDM are acylcarnitines. Acetylcarnitine was increased by $30230 \%$ in the GDM women $(P=0.005)$ as compared to nondiabetic women $(P=0.005)$, whereas 303 long-chain acylcarnitines were reduced by approximately $30 \%(P<0.01)$ in the GDM women. 304 Interestingly, in GDM women carnitine was also reduced by $30 \%$ in plasma $(P=0.0001)$ and by $30555 \%$ in urine $(P=0.02)$. Correlation analyses revealed that plasma acetylcarnitine significantly 306 correlated with 2h-glucose and AUC-G, whereas carnitine and LC-AC showed an inverse 307 correlation with diabetes outcome measures; the highest correlation was found for 308 steaorylcarnitine $(-0.556, P=0.0002 ;-0.574, P=0.0001 ;-0.504, P=0.0009$, for correlation with 309 2h-glucose, AUC-G and HbA1c, respectively). The ratio of long-chain acylcarnitines/carnitine 310 was similar between control and diabetic women (0.38), whereas the acetyl-carnitine/carnitine 311 ratio was significantly augmented in GDM (0.31 and 0.56 in control and GDM, respectively).

312 Finally, we performed a ROC analysis with metabolites that showed the best correlation with 313 diabetes outcome. Only metabolites with a ROC area $>0.94$ are shown in Table 4 . The analysis 314 revealed a high discriminant power for 25 lysoglycerophospholipids, (21 contain a PUFA-chain), 315 arachidonic (20:4) and docosahexaenoic (22:6) acid methylesters, and taurine-conjugated bile 316 acids. Lipoxin was another lipid that exhibited a high discriminant power and also correlated 317 with diabetic outcome. 
320 Despite several recommendations, there is no consensus approach to GDM diagnosis [17]. Thus, 321 the availability of metabolites (or metabolic patterns) that predict GDM would be a major 322 advance. Here, we performed a multiplatform metabolomic analysis of pregnant women at the

$3232^{\text {nd }}$ trimester in order to gain novel insights into the metabolic routes that are specifically altered 324 in GDM and to identify potential biomarkers related to the glucose intolerance of the mother.

325 Metabolomic research in pregnancy has focused mainly on preeclampsia and, to our knowledge, 326 only a few studies have analyzed potential urine biomarkers for GDM [18]. Here, we confirm 327 results of a study on a large multiethnic population, reporting that changes in the urinary 328 excretion profile during and after pregnancy do not yield reliable biomarkers for GDM [19]. In 329 fact, the only alteration we detected in urine samples from GDM women was an increase in the 330 excretion of some aminoacids that did not correlate with glycemic control of the women.

331 On the contrary, individual plasma metabolite fingerprints allowed for a clear discrimination of 332 women with normal glucose tolerance and those with GDM. Identification of these metabolites 333 revealed alterations of various metabolic pathways (for details see Fig. 4). Furthermore, we 334 identified a set of metabolites, the variability of which correlated well with glycemic control and, 335 thus, may provide insights into the metabolic disease etiology.

338 Alterations of the levels and composition of plasma lysophospholipids were the most prominent 339 changes that correlated well with the glycemic state of pregnant women. In particular, LPE(20:1) 340 and (20:2) were affected by GDM and showed the highest discriminant power in the ROC 341 analysis. Data about LPE as bioactive metabolites are scarce as compared to other phospholipids, 
342 although its anti-inflammatory actions has been demonstrated in a mouse model of inflammation

343 [20]. In support of a role for LPE as a biomarker for GDM, a non-targeted metabolomic study

344 showed that $\operatorname{LPE}(16: 1)$ allowed for the classification of subjects as insulin sensitive or insulin

345 resistant [21].

346 We also detected a decrease of various LPIs, in particular those with LC-PUFA(18:2, 20:4,

347 22:6), and of LPC, PC and glycerophosphocholine. We identified LPC(18:2) as one of the

348 metabolites that correlated best with the glycemic control of pregnant women and showed a high

349 discriminative power. Others have reported a decrease in various LPC, PC and 350 glycerophosphocoline in type 2-diabetes [22, 23] and, in various prospective population-based

351 cohort studies, low levels of LPC(18:2) were shown to be predictive for dysglycemia and type 2-

352 diabetes [5, 6]. Interestingly, LPCs, such as LPC(18:2), have been found to induce glucose-

353 induced insulin secretion from pancreatic $\beta$-cells, [5, 24, 25]. Furthermore, LPCs and LPSs were

354 found to improve glycemia in both normal and type 1 and 2 diabetic mice through an enhanced

355 glucose uptake [26, 27]. Thus, the observed decrease of lysoglycerophospholipids in GDM may

356 be associated with glucose intolerance through altered glucose metabolism and $\beta$-cell

357 dysfunction.

358 The observation that GDM was accompanied by a decrease in almost all species of 359 lysoglycerophospholipids, points to an alteration of a common enzymatic activity. Interestingly, 360 patients with IGT and type 2-diabetes were reported to have lower cPLA2 (cytosolic calcium361 dependent phospholipase-A2 isoform) transcription levels [6]. Since PUFAs are typically 362 released from the sn-2 position of phospholipids, reduced cPLA2 activity could account for the 363 decreased concentration of arachidonic acid [6] or other LC-PUFAs associated to type 2-diabetes 
365 Glycerophospholipids with shorter chain length and saturated fatty acid residues may trigger 366 development of type 2-diabetes, whereas those containing LC-PUFAs may offer protection [23, 367 28] and attenuate inflammation induced by saturated acyl LPCs [29, 30], suggesting a role of 368 lysoglycerophospholipids with LC-PUFAs as anti-inflammatory molecules. Interestingly, we

369 found that the ratio of saturated/unsaturated acyl chains in LPCs, LPEs and LPIs was increased 370 in GDM, indicating that lysoglycerophospholipids acyl moieties are determinant in their effect

371 on glucose/lipid metabolism. These results, together with the decrease of lipoxin C4 levels in 372 GDM, suggest that an unbalanced proportion of pro-inflammatory versus anti-inflammatory 373 molecules is characteristic for GDM development. Interestingly, n-6 PUFA-derived lipoxins are 374 potent anti-inflammatory compounds in various models of inflammation and, very recently, it 375 has been proposed that they may act as endogenous anti-diabetic molecules [31].

376 In parallel with the decrease in lysoglycerophospholipids, we observed a decrease of 377 plasmalogens in the GDM group. Various studies report a negative association of 378 glycerophosphocholine-plasmalogens with obesity and insulin resistance [23, 32, 33]. As 379 plasmalogens may act as serum antioxidants to prevent lipoprotein oxidation [33, 34], the 380 decrease that we observed may suggest that low-grade lipid peroxidation occurs already at the 381 beginning of GDM. In fact, in a previous study from our group [35], we found that, in the second 382 trimester of gestation, non-obese women with GDM have already higher plasma concentrations 383 of lipid and protein oxidation products than the control group.

384 We also observed a reduction in various sphingomyelins, ceramide-ethanolamines, and 385 sphingosine 1-phosphate, although no significant differences were found in ceramides between 
control and GDM pregnant women. Sphingolipid metabolism is altered in diabetic conditions but, to date, most studies focused on ceramides [36] and only few examined the involvement of sphingomyelins, obtaining contradictory results. Some studies found a decrease of some sphingomyelins in diabetic patients [22, 23], whereas another study showed an increase [37]. Interestingly, whereas in the former studies, HbA1c was lower than $6.5 \%$, the latter reported that HbA1c was $8.3 \%$. In this context, we found that GDM pregnant women at this stage of gestation had a good glycemic control (HbA1c <5.5\%). Since glucose activates sphingosine kinase, favouring the synthesis of sphingosine 1-phosphate [38], we propose that sphingolipid variations in diabetes are dependent of the metabolic control. Additionally, similar to other complex lipids, the effects of sphingolipids may differ dependent on the acyl moiety. In support of this, type 1diabetes has been associated with a decrease in nervonic acid (24:1), and sphingomyelins-and ceramides containing nervonic acid, whereas those with 20:0 and 24:0 acyl chains are increased [39]. Interestingly, we found a decrease in hydroxy-nervonic acid in GDM, and it has been reported that nervonic acid may have a preventive effect on human metabolic disorders [40]. As detailed in Fig. 4, the observed decrease in sphingomyelins may be related to the partitioning of palmitate to triacylglycerides in a competitive manner [41], favouring the hypertriglyceridemia observed in GDM.

Taurine-conjugated bile acids also exhibited a strong inverse association with the glycemic state and a high discriminating power between control and GDM pregnant women. Novel functions of bile acid as metabolic integrators of energy homeostasis influencing glucose and lipid metabolism have been described, including lowering triacylglycerides, inhibiting 407 gluconeogenesis, and improving insulin sensitivity [42]. In a metabolomic approach performed 408 in the KORA F3 cohort study, it was reported that cholate, a primary bile acid, was detected 
409 more frequently in control subjects than in diabetics, while the opposite was found for 410 deoxycholate, a secondary bile acid [22]. Accordingly, it has been suggested that the bile acid

411 profile is altered in diabetes [43]. Whether the decrease in taurine-conjugated bile acids and/or

412 the altered bile acid pool composition reflect on a metabolic change that could be involved in

413 other GDM metabolic alterations, such as hypertriglyceridemia, or if it is solely discriminating

414 between glucose intolerance under fasting conditions, needs to be examinated.

416 Other metabolites altered in GDM

417 We found that acetyl-carnititine, the main acylcarnitine ester, was increased in GDM, whereas 418 carnitine and long-chain acyl-carnitines were decreased. This increase in acetyl-carnitine in 419 GDM seems to be a common metabolic event of glucose homeostasis alterations, including IGT 420 [6] and diabetes [44]. In fact, increased expression of carnitine acetyl-CoA transferase, the 421 enzyme responsible for acetyl-carnitine synthesis, in blood cells has been reported for IGT and 422 type 2-diabetes [6].

423 An altered fatty acid oxidation has been associated with insulin resistance and diabetes [45]. 424 Interestingly, the early stages of diet-induced insulin resistance, when glucose intolerance but not 425 insulin resistance is present, are characterized by increasing muscle fatty acid oxidation [46]. 426 Furthermore, in different metabolomic studies it was found that medium-chain acylcarnitines 427 decrease with impairing glucose tolerance [21]. The observed correlation of acetyl-carnitine and 428 glucose intolerance tempts us to suggest that, at the beginning of gestation, an increased muscle 429 fatty acid oxidation leads to a decrease in long-chain acylcarnitines, together with a concomitant 430 increase of acetyl-CoA (Fig. 4). In fact, we observed a decrease in carnitine in the GDM group 
431 that seems to be caused by the trapping effect of acetyl-CoA. This is supported by the 432 observation that the ratio of long-chain acylcarnitines/carnitine did not differ between control 433 and diabetic women, whereas the ratio acetyl-carnitine/carnitine was significantly augmented in 434 the second group. Furthermore, the observed decrease in carnitine is considered a hallmark of 435 glucose intolerance and insulin resistance [47].

436 We also found that glycine and pyruvate were significantly reduced in GDM. Recent 437 metabolomics studies found a decrease of glycine in patients with IGT, type 2-diabetes, obesity, 438 and impaired insulin sensitivity [21, 48], and in prospective studies, decreased glycine has also 439 been proposed as an independent predictor of type 2-diabetes [23] and IGT [6]. Reduced glycine 440 in GDM, may reflect on enhanced gluconeogenesis, glutathione synthesis [49] or both (detailed 441 in Fig. 4). The role of glycine as an indicator of increased gluconeogenesis during fasting in the 442 GDM group is further support by the fact that pyruvate is also significantly lower in GDM. It 443 should be considered that, in fasting conditions, pyruvate is used preferentially for 444 gluconeogenesis rather than for oxidation upon conversion to acetyl-CoA. Under these 445 circumstances, fatty acids turn into the predominant susbtrate for energy production. A switch to 446 fatty acid oxidation is further supported by increased levels of 3-hydroxybutyrate in GDM 447 women under fasting conditions (this study), similar to what has been observed previously in the $448 \quad 2^{\text {nd }}$ trimester of gestation in GDM [50].

449 Finally, we observed that plasma 2-hydroxybutyrate levels, an organic acid derived from 2450 ketobutyrate in a reaction catalyzed by lactate dehydrogenase, were higher in GDM than in 451 controls. 2-hydroxybutyrate is also elevated in human and animal models of type 2-diabetes [51] 452 and has been proposed as an independent and early predictor of glucose intolerance in humans 
$453[5,21]$. Accumulation of 2-hydroxybutyrate may occur in vivo when the formation of 2454 ketobutyrate exceeds the rate of its catabolism. As detailed in Fig. 4, our interpretation that a 455 redox imbalance may contribute to elevated 2-hydroxybutyrate is consistent with our finding that 456 fatty acid oxidation is increased in GDM.

457

\section{Concluding remarks}

459 To our knowledge, this study represents the first multi-platform, non-targeted metabolome-wide 460 analyses in plasma and urine of GDM. We show that, in the $2^{\text {nd }}$ trimester of gestation, metabolite 461 fingerprints in plasma reveal metabolic imbalances that are specific for human GDM. Some of 462 the observed alterations have been previously associated with impaired glucose homeostasis. 463 Nonetheless, we were able to identify specific metabolic patterns that are indicative of low-grade 464 inflammation and altered redox-balance, which may reflect on the specific pathophysiological 465 context of GDM. As this is a pilot study, future projects at later stages of gestation will allow us 466 to validate the identified discriminant biomarkers as tools to predict the onset of diabetic 467 complications both during pregnancy and after delivery. 
470 The authors gratefully acknowledge the financial support from Spanish Ministry of Economy

471 and Competitiveness (MINECO- CTQ2011-23562 and SAF2010-19603) and Community of

472 Madrid (S2010/BMD-2423). D.D. acknowledges her postdoc contract with Universidad San

473 Pablo-CEU. No potential conflicts of interest that are relevant to this article were reported. The 474 authors thank all the study participants.

475 D.D. designed the study, performed experiments, analysed data, interpreted results and 476 contributed to edition of the manuscript; M.Z. and G.K. contributed with the collection of 477 samples, biochemical analysis and data acquisition; W.Z. contributed to biochemical analyis; 478 M.S. and K.B-M. provided clinical data and expertise in clinical interpretation; M.V. and A.G. 479 performed research and contributed to data analysis; C.B. is the guarantor of this work, 480 contributed to the supervision of experiments and data interpretation; MP.R performed data 481 analysis, coordinated data interpretation and wrote the manuscript. All authors reviewed and 482 accepted the manuscript.

483 Part of this study was presented at the 49th Annual EASD Meeting (September 23-27, 2013) and 484 at the 9th International Conference of the Metabolomics Society (July 1-4, 2013).

485 DISCLOSURES

486 No conflicts of interest, financial or otherwise, are declared by the authors. 
[1] Association AD. Diagnosis and classification of diabetes mellitus. Diabetes Care. 2009;32 Suppl 1:S62-7.

[2] International Association of D, Pregnancy Study Groups Consensus P, Metzger BE, Gabbe SG, Persson B, Buchanan TA, et al. International association of diabetes and pregnancy study groups recommendations on the diagnosis and classification of hyperglycemia in pregnancy. Diabetes Care. 2010;33:676-82.

[3] Lowe LP, Metzger BE, Dyer AR, Lowe J, McCance DR, Lappin TR, et al. Hyperglycemia and Adverse Pregnancy Outcome (HAPO) Study: associations of maternal A1C and glucose with pregnancy outcomes. Diabetes Care. 2012;35:574-80.

[4] Bellamy L, Casas JP, Hingorani AD, Williams D. Type 2 diabetes mellitus after gestational diabetes: a systematic review and meta-analysis. Lancet. 2009;373:1773-9.

[5] Ferrannini E, Natali A, Camastra S, Nannipieri M, Mari A, Adam KP, et al. Early metabolic markers of the development of dysglycemia and type 2 diabetes and their physiological significance. Diabetes. 2013;62:1730-7.

504 pre-diabetes identified by metabolomics. Mol Syst Biol. 2012;8:615.

[7] Lowe WL, Jr., Bain JR. "Prediction is very hard, especially about the future": new biomarkers for type 2 diabetes? Diabetes. 2013;62:1384-5.

508 [8] Matthews DR, Hosker JP, Rudenski AS, Naylor BA, Treacher DF, Turner RC. Homeostasis model assessment: insulin resistance and beta-cell function from fasting plasma glucose and insulin concentrations in man. Diabetologia. 1985;28:412-9.

[9] Katz A, Nambi SS, Mather K, Baron AD, Follmann DA, Sullivan G, et al. Quantitative insulin sensitivity check index: a simple, accurate method for assessing insulin sensitivity in humans. J Clin Endocrinol Metab. 2000;85:2402-10.

[10] Ciborowski M, Javier Ruperez F, Martinez-Alcazar MP, Angulo S, Radziwon P, Olszanski $\mathrm{R}$, et al. Metabolomic approach with LC-MS reveals significant effect of pressure on diver's plasma. J Proteome Res. 2010;9:4131-7.

[11] Vallejo M, Garcia A, Tunon J, Garcia-Martinez D, Angulo S, Martin-Ventura JL, et al. Plasma fingerprinting with GC-MS in acute coronary syndrome. Anal Bioanal Chem. 2009;394:1517-24.

[12] Moraes EP, Ruperez FJ, Plaza M, Herrero M, Barbas C. Metabolomic assessment with CEMS of the nutraceutical effect of Cystoseira spp extracts in an animal model. Electrophoresis. 2011;32:2055-62.

[13] Gika HG, Macpherson E, Theodoridis GA, Wilson ID. Evaluation of the repeatability of ultra-performance liquid chromatography-TOF-MS for global metabolic profiling of human urine samples. J Chromatogr B Analyt Technol Biomed Life Sci. 2008;871:299-305.

528 [15] Dunn WB, Broadhurst D, Begley P, Zelena E, Francis-McIntyre S, Anderson N, et al. 529 Procedures for large-scale metabolic profiling of serum and plasma using gas chromatography 530 
[16] Westerhuis J, Hoefsloot HJ, Smit S, Vis D, Smilde A, Velzen EJ, et al. Assessment of PLSDA cross validation. Metabolomics. 2008;4:81-9.

[17] Weinert LS. International Association of Diabetes and Pregnancy Study Groups recommendations on the diagnosis and classification of hyperglycemia in pregnancy: comment to the International Association of Diabetes and Pregnancy Study Groups Consensus Panel. Diabetes Care. 2010;33:e97; author reply e8.

[18] Fanos V, Atzori L, Makarenko K, Melis GB, Ferrazzi E. Metabolomics application in maternal-fetal medicine. Biomed Res Int. 2013;2013:720514.

[19] Sachse D, Sletner L, Morkrid K, Jenum AK, Birkeland KI, Rise F, et al. Metabolic changes in urine during and after pregnancy in a large, multiethnic population-based cohort study of gestational diabetes. PLoS One. 2012;7:e52399.

[20] Hung ND, Kim MR, Sok DE. 2-Polyunsaturated acyl lysophosphatidylethanolamine attenuates inflammatory response in zymosan A-induced peritonitis in mice. Lipids. 2011;46:893-906.

[21] Gall WE, Beebe K, Lawton KA, Adam KP, Mitchell MW, Nakhle PJ, et al. alphahydroxybutyrate is an early biomarker of insulin resistance and glucose intolerance in a nondiabetic population. PLoS One. 2010;5:e10883.

[22] Suhre K, Meisinger C, Doring A, Altmaier E, Belcredi P, Gieger C, et al. Metabolic footprint of diabetes: a multiplatform metabolomics study in an epidemiological setting. PLoS One. 2010;5:e13953.

[23] Floegel A, Stefan N, Yu Z, Muhlenbruch K, Drogan D, Joost HG, et al. Identification of serum metabolites associated with risk of type 2 diabetes using a targeted metabolomic approach. Diabetes. 2013;62:639-48.

[24] Soga T, Ohishi T, Matsui T, Saito T, Matsumoto M, Takasaki J, et al. Lysophosphatidylcholine enhances glucose-dependent insulin secretion via an orphan G-proteincoupled receptor. Biochem Biophys Res Commun. 2005;326:744-51.

[25] Metz SA. Ether-linked lysophospholipids initiate insulin secretion. Lysophospholipids may mediate effects of phospholipase A2 activation on hormone release. Diabetes. 1986;35:808-17.

[26] Yea K, Kim J, Yoon JH, Kwon T, Kim JH, Lee BD, et al. Lysophosphatidylcholine activates adipocyte glucose uptake and lowers blood glucose levels in murine models of diabetes. J Biol Chem. 2009;284:33833-40.

[27] Yea K, Kim J, Lim S, Kwon T, Park HS, Park KS, et al. Lysophosphatidylserine regulates blood glucose by enhancing glucose transport in myotubes and adipocytes. Biochem Biophys Res Commun. 2009;378:783-8.

[28] Kroger J, Zietemann V, Enzenbach C, Weikert C, Jansen EH, Doring F, et al. Erythrocyte membrane phospholipid fatty acids, desaturase activity, and dietary fatty acids in relation to risk of type 2 diabetes in the European Prospective Investigation into Cancer and Nutrition (EPIC)Potsdam Study. The American journal of clinical nutrition. 2011;93:127-42.

[29] Riederer M, Ojala PJ, Hrzenjak A, Graier WF, Malli R, Tritscher M, et al. Acyl chaindependent effect of lysophosphatidylcholine on endothelial prostacyclin production. J Lipid Res. 2010;51:2957-66.

[30] Hung ND, Sok DE, Kim MR. Prevention of 1-palmitoyl lysophosphatidylcholine-induced inflammation by polyunsaturated acyl lysophosphatidylcholine. Inflamm Res. 2012;61:473-83. [31] Das UN. Arachidonic acid and lipoxin A4 as possible endogenous anti-diabetic molecules. Prostaglandins Leukot Essent Fatty Acids. 2013;88:201-10. 
[32] Pietilainen KH, Sysi-Aho M, Rissanen A, Seppanen-Laakso T, Yki-Jarvinen H, Kaprio J, et al. Acquired obesity is associated with changes in the serum lipidomic profile independent of genetic effects--a monozygotic twin study. PLoS One. 2007;2:e218.

[33] Wallner S, Schmitz G. Plasmalogens the neglected regulatory and scavenging lipid species. Chem Phys Lipids. 2011;164:573-89.

[34] Engelmann B. Plasmalogens: targets for oxidants and major lipophilic antioxidants. Biochem Soc Trans. 2004;32:147-50.

[35] Ramos Alvarez MP, Viana M, Alcalá Díaz-Mor M, Bolado VE, Pita Santibáñez J, Espino $\mathrm{M}$, et al. Gestational diabetes and obesity: role of oxidative stress and inflammation. FEBS Lett. 2012;279

[36] Deevska GM, Nikolova-Karakashian MN. The twists and turns of sphingolipid pathway in glucose regulation. Biochimie. 2011;93:32-8.

[37] Zhu C, Liang QL, Hu P, Wang YM, Luo GA. Phospholipidomic identification of potential plasma biomarkers associated with type 2 diabetes mellitus and diabetic nephropathy. Talanta. 2011;85:1711-20.

[38] Wang L, Xing XP, Holmes A, Wadham C, Gamble JR, Vadas MA, et al. Activation of the sphingosine kinase-signaling pathway by high glucose mediates the proinflammatory phenotype of endothelial cells. Circ Res. 2005;97:891-9.

[39] Fox TE, Bewley MC, Unrath KA, Pedersen MM, Anderson RE, Jung DY, et al. Circulating sphingolipid biomarkers in models of type 1 diabetes. J Lipid Res. 2011;52:509-17.

[40] Oda E, Hatada K, Kimura J, Aizawa Y, Thanikachalam PV, Watanabe K. Relationships between serum unsaturated fatty acids and coronary risk factors: negative relations between nervonic acid and obesity-related risk factors. Int Heart J. 2005;46:975-85.

[41] Watson ML, Coghlan M, Hundal HS. Modulating serine palmitoyl transferase (SPT) expression and activity unveils a crucial role in lipid-induced insulin resistance in rat skeletal muscle cells. Biochem J. 2009;417:791-801.

[42] Lefebvre P, Cariou B, Lien F, Kuipers F, Staels B. Role of bile acids and bile acid receptors in metabolic regulation. Physiol Rev. 2009;89:147-91.

[43] Staels B, Kuipers F. Bile acid sequestrants and the treatment of type 2 diabetes mellitus. Drugs. 2007;67:1383-92.

[44] Adams SH, Hoppel CL, Lok KH, Zhao L, Wong SW, Minkler PE, et al. Plasma Acylcarnitine Profiles Suggest Incomplete Long-Chain Fatty Acid $\beta$-Oxidation and Altered Tricarboxylic Acid Cycle Activity in Type 2 Diabetic African-American Women. J Nutr. 2009;139:1073-81.

[45] Schooneman MG, Vaz FM, Houten SM, Soeters MR. Acylcarnitines: reflecting or inflicting insulin resistance? Diabetes. 2013;62:1-8.

[46] Trajcevski KE, O'Neill HM, Wang DC, Thomas MM, Al-Sajee D, Steinberg GR, et al. Enhanced lipid oxidation and maintenance of muscle insulin sensitivity despite glucose intolerance in a diet-induced obesity mouse model. PLoS One. 2013;8:e71747.

[47] Poorabbas A, Fallah F, Bagdadchi J, Mahdavi R, Aliasgarzadeh A, Asadi Y, et al. Determination of free L-carnitine levels in type II diabetic women with and without complications. Eur J Clin Nutr. 2007;61:892-5.

[48] Wurtz P, Makinen VP, Soininen P, Kangas AJ, Tukiainen T, Kettunen J, et al. Metabolic signatures of insulin resistance in 7,098 young adults. Diabetes. 2012;61:1372-80. 
620 [49] Sekhar RV, McKay SV, Patel SG, Guthikonda AP, Reddy VT, Balasubramanyam A, et al. 621 Glutathione synthesis is diminished in patients with uncontrolled diabetes and restored by dietary 622 supplementation with cysteine and glycine. Diabetes Care. 2011;34:162-7.

623 [50] Montelongo A, Lasuncion MA, Pallardo LF, Herrera E. Longitudinal study of plasma 624 lipoproteins and hormones during pregnancy in normal and diabetic women. Diabetes. 625 1992;41:1651-9.

626 [51] Salek RM, Maguire ML, Bentley E, Rubtsov DV, Hough T, Cheeseman M, et al. A 627 metabolomic comparison of urinary changes in type 2 diabetes in mouse, rat, and human. Physiol 628 Genomics. 2007;29:99-108. 
Figure 1. Score plots of plasma (A-E) and urine (F) metabolic profiles obtained for control $(\square)$ and GDM women (O).

\section{A-B. Unsupervised PCA analysis.}

(A) LC-MS ESI+ $\left(\mathrm{R}^{2}=0.53, \mathrm{Q}^{2}=0.48\right)$ (B) LC-MS ESI- $\left(\mathrm{R}^{2}=0.43, \mathrm{Q}^{2}=0.34\right)$. The plots indicate that healthy controls can be clearly separated from most of GDM individuals.

\section{C-F. Supervised OPLS/O2PLS-DA analysis.}

C, D- OPLS/O2PLS-DA model $\left(\mathrm{R}^{2}=0.97, \mathrm{Q}^{2}=0.83\right.$ and $\left.\mathrm{R}^{2}=0.99, \mathrm{Q}^{2}=0.9\right)$ built for the two groups (GDM women versus healthy controls) based on 626 LC-MS ESI+ (C) and 487 LC-MS ESI- (D) detected variables.

E, F- OPLS/O2PLS-DA model built for the two groups according to 48 GC-MS $\left(\mathrm{R}^{2}=0.75\right.$, $\left.\mathrm{Q}^{2}=0.5\right)(\mathrm{E})$ and $127 \mathrm{CE}-\mathrm{MS}\left(\mathrm{R}^{2}=0.91, \mathrm{Q}^{2}=0.69\right)(\mathrm{F})$ detected variables.

$\mathrm{R}^{2}=$ coefficient for variance explained; $\mathrm{Q}^{2}=$ coefficient for variance predicted.

\section{Figure 2. Dendrogram and heat map of plasma metabolites.}

Cluster analysis of LC-MS data was performed in order to identify patterns of metabolites that discriminate between control and GDM women. The heat map represents the signal intensities of 20 pregnant women with normal glucose tolerance $(\square)$, and 20 pregnant women that were diagnosed with GDM ( $\square$ ) according to the 2h-OGTT. Colors reflect on signal intensity; measured in plasma samples. The spectrum ranging from red to blue represents the range of high to low signal intensities, respectively, for each metabolite, identified by numbers as described below. Black color indicates missing values. The $\mathrm{X}$-axis was divided into two sections by a white discontinuous line, representing $2 \mathrm{~h}$-glucose below or above the threshold value of $>140$ $\mathrm{mg} / \mathrm{dL}(7,8 \mathrm{mM})$ for diagnosis of GDM. The Y-axis was divided into three sections, representing high, medium and low metabolite concentrations (the identification of all compounds is available as supplementary material). Two samples, classified as control (17C) and GDM (20D) according to the 2h-OGTT, are marked with arrows to highlight a different metabolic pattern of their corresponding group. Follow-up of these women throughout pregnancy revealed that, two weeks before delivery, the study participant 17C who was classified as control had HbA1c of 9\%. The GDM woman 20D received dietary treatment and, during the rest of gestation, fasting glucose fell below $100 \mathrm{mg} / \mathrm{dL}(5.55 \mathrm{mM})$.

1-SM(34:1); 2-Oleamide; 3-LPC(16:0); 4-SM(36:2); 5-SM(34:2); 6-LPC(18:2)sn-2; 7-Linoleamide; 8Palimitic amide; 9-LPA(16:0); 10-Dodecanamide; 11-LPI(16:1); 12-LPI(16:0); 13-PC(40:3); 14PC(38:1); 15-PC(32:2); 16-PE(36:3); 17-LPC(18:1)sn-2; 18-LPC(18:0)sn-2; 19-PC(36:6); 20Docosenamide; 21-PC(35:4); 22-PC(O-38:6) or PC(P-38:5); 23-LPE(20:1); 24-LPE(20:0); 25-LPE(18:0); 
686

687

688

689

690

691

692

693

694

695

696

697

698

699

700

701

26-LPC(18:3)sn-2; 27-LPE(20:2); 28-Oleoyl Ethylamide; 29-Octadecatrienal; 30-PE(38:5); 31-PC(40:9); 32-PC(40:7); 33-Octadecatrienol; 34-PC(36:5); 35-PC(40:8); 36-PC(38:7); 37-LPE(16:0); 38-SM(36:3); 39-PC(38:5); 40-LPE(22:6); 41-LPE(22:4); 42-Asparylthreonine; 43-Acetylglutamine; 44-Phosphatidylmyo-inositol; 45-PC(36:4); 46-LPE(20:4); 47-PC(P-36:5); 48-LPE(18:1); 49-LPE(O-18:1) or LPE(P18:0); 50-LPI(18:2); 51-LPC(22:5)sn-2; 52-LPC(20:4)sn-2; 53-LPC(17:0)sn-2; 54-LPC(O-16:0)sn-1; 55Acetylcarnitine; 56-PC(42:7); 57-LPC (20:3)sn-1; 58-LPI(20:4); 59-LPS(20:0); 60-LPC(20:5)sn-2; 61Lipoxin C4; 62-LPE(18:2); 63-LPC(16:1)sn-2; 64-SM(32:2); 65-Hexadecatrienol; 66-Trioxocholenoic acid; 67-PC(35:3); 68-Carnitine; 69-Taurolithocholic acid glucuronide; 70-PC(19:1); 71-PE(38:6); 72$\mathrm{PE}(36: 5)$; 73-Asparylhydrohyproline; 74-Cerebronic acid; 75-PS(26:0); 76-PC(21:0 (COOH)); 77LPI(20:3); 78-LPC(22:6)sn-2; 79-LPC(22:4)sn-2; 80-Oxo-nonadecanoic acid; 81-LPS(20:2); 82Hydroxynervonic acid; 83-LPE(19:0); 84-LPA(18:2); 85-LPE(18:3); 86-LPE(22:1); 87-LPC(O-18:1)sn-1 or LPC (P-18:O)sn-1; 88-PC(27:0); 89-LPC(19:1); 90-Stearoylcarnitine; 91-Linoleylcarnitine; 92PC(42:9); 93-SM(33:2); 94-Trihydroxy-cholestanoyl taurine; 95-LPC(20:2)sn-2; 96Glycerophosphocholine; 97-Arachidonic Acid Methylester; 98-Docosahexaenoic Acid Methylester; 99PC(21:1); 100-PC(42:8); 101-Dihydroxytetranorvitamin D3; 102-Pentadecadienal; 103-PC(19:0); 104LPI(22:6); 105-Anandamide (20:2, n-6); 106-LPE(P-20:0); 107- LPC(20:0)sn-2; 108-LPC(17:1)sn-2; 109-LPC(24:0)sn-2; 110-LPC(O-18:0)sn-1; 111-Dimethyl-undecadienone; 112-Tetradecadienal; 113Tetramethyltridecadienal; 114-Dodecadienal; 115-Oxo-heneicosanoic acid; 116-Sphingosine-phosphate; 117-Palmitoylcarnitine; 118-PA(28:0); 119-PA(38:6); 120-LPC(14:0)sn-2; 121-Epoxy-dimethylcyclocholestan-ol; 122-PE-Cer(34:1); 123-LPE(P-16:0); 124-LPC(20:1)sn-1; 125-Palmitoyl Isopropylamide; 126-LPC(15:0)sn-2; 127-PE-Cer(33:1); 128-Vaccenylcarnitine; 129-PC(42:10); 130Hydroxy-oxo-cholanoic acid; 131-Histidine.

Figure 3. Comparative description of the main lysoglycerophospholipids in control and GDM pregnant women at the second trimester of gestation.

(A) lysophosphatidylcholines (LPC); (B) lysophosphatidylethanolamines (LPE) and (C) lysophosphatidylinositols (LPI).

Data are given in percentage (\%) and represent the area of the lysoglycerophospholipids with saturated, monounsaturated (MUFA) or polyunsaturated (PUFA) acyl chains relative to the total area.

Figure 4. Proposed model of metabolic alterations in the second trimester of GDM, with a special focus on lipid metabolism.

We hypothesized that, at beginning of pregnancy, GDM is characterized by an increased response to fasting. During this response, fatty acids (NEFA) turn into the major substrate for energy production, favouring oxidative stress and a mild inflammatory condition. In this scenario, an enhanced lipolysis (1) in adipose tissue (supported by increased glycerol and linoleic acid (C18:2)) favors liver and muscle NEFA availability. In both tissues, lipid overload drives an 
intramitochondrial flux of acyl-CoA for NEFA oxidation, which results in decreased long-chain acylcarnitines (LC-AC) and accumulation of acetyl-CoA (2). This metabolite is converted into acetyl-carnitine (3), permitting its mitochondrial efflux that otherwise would inhibit pyruvate dehydrogenase. This situation causes depletion of carnitine and, consequently, decreased excretion of this metabolite into the circulation as well as increased levels of circulating acetylcarnitine (4).

In humans, the liver accounts for most of the NEFA oxidation during fasting. In this condition, acetyl-CoA can be used for 3-hydroxybutyrate (3-HB) synthesis, contributing to the ketonemia observed in GDM women (5), or may activate pyruvate carboxylase, favoring gluconeogenesis (6). Thus, pyruvate and glycerol (7) are used preferentially for gluconeogenesis, favouring glucose intolerance (8). Reduced glycine in GDM may also reflect on enhanced gluconeogenesis, since glycine can be converted to glucose via pyruvate production or/and, to glutathione (GSH) (9).

Enhanced mitochondrial activity also increases the $\mathrm{NADH}+\mathrm{H}^{+} / \mathrm{NAD}^{+}$ratio and oxygen radical production (2). To cope with the resulting oxidative stress, glutathione biosynthesis is activated (10), which is supported by the observed decreased in glycine and glutamate and the concomitant increase of 2-hydroxybutyrate (2-HB). 2-ketobutyrate (2-KB) is produced through the conversion of cystathionine to cysteine for glutathione biosynthesis (11). Subsequently, 2-KB is reduced to $2-\mathrm{HB}(\mathbf{1 2})$, which is favored by the observed increase of the NADH/NAD ${ }^{+}$ratio (2). Thus, $2-\mathrm{HB}$ is associated with an increased demand for glutathione biosynthesis and disrupted mitochondrial energy metabolism.

The reduced de novo sphingolipid synthesis found in GDM, probably as a consequence of serine availability (13), favors the flux of palmitate (16:0) towards TG, leading to the hypertrigliceridemia observed in GDM women (14). In this condition, enhanced TG biosynthesis may also cause that phosphatidic acid is not used for the synthesis of glycerophospholipids (PL) (15), favouring the observed decrease in lysophospholipids (LysoPL). The fact that cysteine metabolism is favoring glutathione biosynthesis may be associated with a decrease in SAM, a key molecule for the transformation of PC to PE in the liver.

Finally, de novo sphingolipid synthesis is reduced (13); probably ceramides are synthesized via sphingomyelin hydrolysis or through the salvage pathway from sphingosine 1-phosphate (S1P) (16), which would explain the observed decrease of these lipids. Decreased ethanolamineplasmalogen (Et-Plasm) levels may be a consequence of their increased utilization as antioxidants (17) or of a decreased synthesis from $\mathrm{S} 1 \mathrm{P}(\mathbf{1 8})$.

\section{Other abbreviations: OAA: oxaloacetate; 2-KG: 2-ketoglutarate.}

Arrows indicate whether the level of a given metabolite was increased (red) or decreased (blue) according to the metabolome analysis performed in the present study Discontinuous arrows represent a reduced utilization of the corresponding metabolic route. 
$739 *$ gluconeogenesis takes place only in the liver. 
$2^{\text {nd }}$ Trimester Pregnant Women
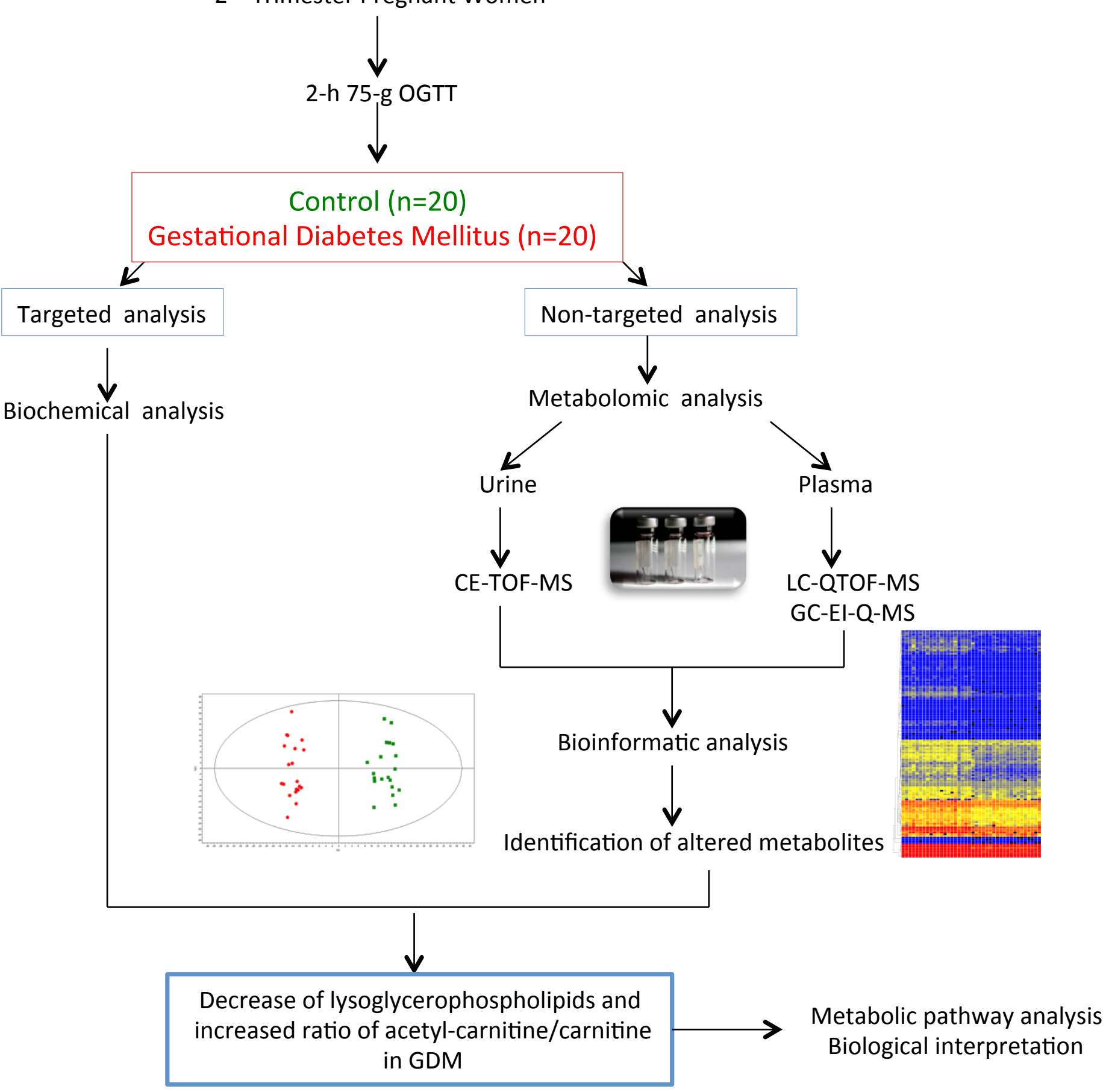
- First multiplatform non-targeted metabolomic analysis of human gestational diabetes.

- Plasma fingerprints reveal disease-specific metabolic imbalances in diabetic women.

- Lysoglycerophospholipids reflect on glucose intolerance in gestational diabetes. 
Table 1. Anthropometric and metabolic characteristic of the women included in the study.

\begin{tabular}{|c|c|c|c|}
\hline Parameter & $\begin{array}{c}\text { Control group } \\
\mathbf{n}=20\end{array}$ & $\begin{array}{c}\text { GDM group } \\
\mathbf{n}=\mathbf{2 0}\end{array}$ & $P$-value \\
\hline Age (years) & $28.5 \pm 2.7$ & $28.1 \pm 4.7$ & ns \\
\hline Parity (number) & $1.3 \pm 0.6$ & $1.4 \pm 0.8$ & ns \\
\hline Week of gestation & $24.8 \pm 1.3$ & $25.5 \pm 1.6$ & ns \\
\hline Pre-pregnancy BMI $\left(\mathrm{kg} / \mathrm{m}^{2}\right)$ & $22.0 \pm 2.7$ & $24.6 \pm 5.1$ & ns \\
\hline Pregnancy BMI $\left(\mathrm{kg} / \mathrm{m}^{2}\right)$ & $23.8 \pm 2.5$ & $27.4 \pm 5.5$ & 0.01 \\
\hline Fasting glucose (mmol/L) & $4.41 \pm 0.29$ & $5.10 \pm 0.79$ & 0.001 \\
\hline 1-h glucose, OGTT (mmol/L) & $6.64 \pm 0.91$ & $8.99 \pm 1.87$ & $<0.0001$ \\
\hline 2-h glucose, OGTT (mmol/L) & $5.76 \pm 0.91$ & $8.97 \pm 1.02$ & $<0.0001$ \\
\hline AUC-G & $12680 \pm 1316$ & $17324 \pm 2204$ & $<0.0001$ \\
\hline $\mathrm{HbA1}_{\mathrm{C}}$ & $\begin{array}{c}4.78 \pm 0.31 \\
28.7 \pm 3.4\end{array}$ & $\begin{array}{c}5.23 \pm 0.39 \\
33.6 \pm 4.3\end{array}$ & 0.0003 \\
\hline Insulin (pmol/L) & $73.8 \pm 22.0$ & $78.5 \pm 35.5$ & ns \\
\hline C Peptide (pmol/L) & $0.53 \pm 0.16$ & $0.58 \pm 0.21$ & ns \\
\hline HOMA-IR & $2.43 \pm 0.78$ & $2.98 \pm 1.44$ & ns \\
\hline QUICKI & $0.34 \pm 0.02$ & $0.33 \pm 0.02$ & ns \\
\hline Triacylglycerides (mmol/L) & $1.58 \pm 0.60$ & $2.19 \pm 0.64$ & 0.004 \\
\hline Total cholesterol (mmol/L) & $6.30 \pm 0.95$ & $6.94 \pm 0.92$ & 0.038 \\
\hline LDL-cholesterol (mmol/L) & $3.51 \pm 0.76$ & $3.75 \pm 0.91$ & ns \\
\hline HDL-cholesterol (mmol/L) & $2.13 \pm 0.45$ & $2.23 \pm 0.42$ & ns \\
\hline CRP $(\mu \mathrm{g} / \mathrm{ml})$ & $3.94 \pm 3.29$ & $4.94 \pm 3.94$ & $\mathrm{~ns}$ \\
\hline Systolic blood pressure (mmHg) & $117.0 \pm 7.2$ & $117.0 \pm 11.8$ & ns \\
\hline Diastolic blood pressure (mmHg) & $72.3 \pm 9.6$ & $71.6 \pm 9.6$ & ns \\
\hline
\end{tabular}

Presented data are mean \pm SD. Statistical comparisons assuming equal ( $t$ test) or unequal variance (Welch's $t$ test) were performed as appropriate. AUC-G: Area under the curve of glucose during the OGTT. Results were considered significant when $P<0.05$. 
Table 2. List of selected metabolites identified in plasma or urine by a multiplatform metabolomic analysis that exhibit the most significant changes with gestational diabetes.

\begin{tabular}{|c|c|c|c|}
\hline Identified compound & $P$ value & $\begin{array}{c}\text { Change } \\
(\%)\end{array}$ & $\begin{array}{c}\mathrm{CV} \text { in QC } \\
(\%)\end{array}$ \\
\hline \multicolumn{4}{|c|}{ LC-MS (Plasma) } \\
\hline LPE(20:2) & $6.66 \mathrm{E}-15$ & -66 & 7 \\
\hline LPE(20:1) & $2.08 \mathrm{E}-14$ & -59 & 4 \\
\hline Trihydroxy-cholestanoyl taurine & $9.70 \mathrm{E}-14$ & -53 & 5 \\
\hline LPA(18:2) & $1.54 \mathrm{E}-13$ & -56 & 18 \\
\hline LPC(20:5) sn-2 & $8.27 \mathrm{E}-13$ & -73 & 14 \\
\hline LPI(20:4) & $1.43 \mathrm{E}-12$ & -75 & 8 \\
\hline LPC(18:2) sn-2 & $1.72 \mathrm{E}-12$ & -66 & 15 \\
\hline $\mathbf{P C}(21: 1)$ & $1.72 \mathrm{E}-12$ & -77 & 23 \\
\hline LPC(18:1) sn-2 & $5.32 \mathrm{E}-12$ & -63 & 11 \\
\hline LPE(22:4) & $5.66 \mathrm{E}-12$ & -59 & 6 \\
\hline LPS(20:0) & $6.07 \mathrm{E}-12$ & -63 & 7 \\
\hline Lipoxin C4 & $8.26 \mathrm{E}-12$ & -39 & 10 \\
\hline LPC(22:5) sn-2 & $9.77 \mathrm{E}-12$ & -70 & 12 \\
\hline LPC(22:4) sn-2 & $1.67 \mathrm{E}-11$ & -69 & 16 \\
\hline LPI(18:2) & $2.38 \mathrm{E}-11$ & -73 & 9 \\
\hline LPC(20:2) sn-2 & $2.60 \mathrm{E}-11$ & -67 & 15 \\
\hline LPC(20:4) sn-2 & $3.55 \mathrm{E}-11$ & -76 & 12 \\
\hline Taurolithocholic acid glucuronide & $4.93 \mathrm{E}-11$ & -60 & 23 \\
\hline LPC(19:1) & $1.90 \mathrm{E}-10$ & -54 & 11 \\
\hline Glycerophosphocholine & $3.22 \mathrm{E}-10$ & -61 & 4 \\
\hline Docosahexaenoic acid Methylester & $3.25 \mathrm{E}-10$ & -73 & 11 \\
\hline LPI(22:6) & $4.66 \mathrm{E}-10$ & -70 & 26 \\
\hline Arachidonic acid Methylester & $7.76 \mathrm{E}-10$ & -72 & 19 \\
\hline LPC(22:6) sn-2 & $1.02 \mathrm{E}-09$ & -55 & 9 \\
\hline LPI(20:3) & $1.86 \mathrm{E}-09$ & -67 & 20 \\
\hline LPE(18:2) & 4.61E-08 & -51 & 5 \\
\hline LPE(20:4) & $6.25 \mathrm{E}-08$ & -52 & 7 \\
\hline
\end{tabular}




\begin{tabular}{|c|c|c|c|}
\hline LPE(22:6) & $6.40 \mathrm{E}-08$ & -44 & 4 \\
\hline \multicolumn{4}{|c|}{ GC-MS (Plasma) } \\
\hline Creatinine & $3.1 \mathrm{E}-5$ & -49 & 10 \\
\hline Pyruvic acid & $5.0 \mathrm{E}-5$ & -54 & \\
\hline L-tryptophan & $2.0 \mathrm{E}-3$ & -24 & 25 \\
\hline 2-hydroxybutyric acid & $3.3 \mathrm{E}-3$ & 68 & 13 \\
\hline Glycine & $6.2 \mathrm{E}-3$ & -39 & 15 \\
\hline L-glutamic acid & $1.7 \mathrm{E}-2$ & -14 & 11 \\
\hline Lauric acid & $1,9 \mathrm{E}-2$ & -24 & 8 \\
\hline Glycerol & $3.4 \mathrm{E}-2$ & 19 & 14 \\
\hline 3-hydroxybutyric acid & $5.0 \mathrm{E}-2$ & 75 & \\
\hline Linoleic acid & $5.0 \mathrm{E}-2$ & 19 & 11 \\
\hline Fumaric acid & $5.0 \mathrm{E}-2$ & 15 & \\
\hline \multicolumn{4}{|c|}{ CE-MS (Urine) } \\
\hline Carnitine & $2.99 \mathrm{E}-02$ & -46 & 12 \\
\hline Histidine & $3.20 \mathrm{E}-02$ & 32 & 4 \\
\hline Glutamine & $\mathrm{JK}$ & 36 & 15 \\
\hline Phenylalanine & $\mathrm{JK}$ & 19 & 4 \\
\hline Tryptophan & $\mathrm{JK}$ & 23 & 4 \\
\hline Cystine & JK & 24 & 7 \\
\hline
\end{tabular}

For LC-MS only those metabolites with $P$ values $<1.10^{-7}$ are shown. The complete list of compounds is included as supplementary material. For GC-MS and CE-MS all compounds with $P<0.05$ between GDM and controls are shown. \% change represents the increase $(+)$ or decrease (-) of the mean in the gestational diabetes group with respect to the control group, the sign indicates the direction of the change. CV in QC indicates the $\%$ of variation of the quality control that was included in the analysis. When necessary data were transformed by applying a $\log$ (base 2) in order to approximate a normal distribution. Univariate statistical analysis assuming equal ( $t$ test) or unequal variance (Welch's $t$ test) were performed as appropriate. $P$ value was corrected according FDR test and $P<0.05$ was considered significant. Multivariate statistical analysis Jack-Knife (JK) confidence intervals estimative, 95\% confidence level. 
Table 3. Correlation analysis.

\begin{tabular}{|c|c|c|c|c|c|}
\hline Compound & Oh glucose & 2h Glucose & AUC-G & $\mathbf{H B A}_{1 \mathrm{C}}$ & HOMA \\
\hline Arachidonic acid methylester & $-0.4152 * *$ & $-0.7984^{\# \# \#}$ & $-0.7331^{\# \# \#}$ & $-0.5228 * * *$ & -0.1974 \\
\hline $\operatorname{LPS}(21: 0)$ & $-0.3342 *$ & $-0.7971^{\# \# \#}$ & $-0.6743^{\# \#}$ & $-0.5052 * * *$ & -0.1353 \\
\hline LPE(20:1) & $-0.3410^{*}$ & $-0.7934^{\# \# \#}$ & $-0.6720^{\# \#}$ & $-0.5076^{* * *}$ & -0.1447 \\
\hline $\begin{array}{l}\text { Trihydroxy-cholestanoyl } \\
\text { taurine }\end{array}$ & $-0.3374 *$ & $-0.7893^{\# \# \#}$ & $-0.6631^{\#}$ & $-0.4908 * *$ & -0.1519 \\
\hline LPE $(20: 2)$ & $-0.3869 *$ & $-0.7812^{\# \# \#}$ & $-0.6799^{\# \#}$ & $-0.5394 * *$ & -0.0712 \\
\hline LPC(18:2)sn-2 & $-0.3984 *$ & $-0.7713^{\# \# \#}$ & $-0.6893^{\# \#}$ & $-0.5530 * *$ & -0.1790 \\
\hline LPC(20:4)sn-2 & $-0.3391 *$ & $-0.7684^{\# \# \#}$ & $-0.6691^{\# \#}$ & $-0.4808 * *$ & -0.2090 \\
\hline LPC(18:1)sn-2 & $-0.3183^{*}$ & $-0.7658^{\# \# \#}$ & $-0.6392^{\# \#}$ & $-0.4753^{* *}$ & -0.1664 \\
\hline LPI(18:2) & $-0.3804 *$ & $-0.7649^{\# \# \#}$ & $-0.6577^{\# \#}$ & $-0.5348 * * *$ & -0.1112 \\
\hline LPS(20:0) & $-0.3909 *$ & $-0.7633^{\# \# \#}$ & $-0.7062^{\# \# \#}$ & $-0.5194 * * *$ & -0.1006 \\
\hline LPI(20:4) & $-0.3675^{*}$ & -0.7576 & $-0.6762^{\# \#}$ & $-0.4601 * *$ & -0.0846 \\
\hline LPC(20:5)sn-2 & $-0.4028 * *$ & $-0.7531^{\# \# \#}$ & $-0.6801^{\# \#}$ & $-0.5204 * * *$ & -0.2019 \\
\hline $\begin{array}{l}\text { Taurolithocholic acid } \\
\text { glucuronide }\end{array}$ & $-0.3344 *$ & $-0.7489^{\# \# \#}$ & $-0.6392^{\#}$ & $-0.4117 * *$ & $-0,1121$ \\
\hline LPE(22:4) & $-0.3619 *$ & $-0.7472^{\# \# \#}$ & $-0.6936^{\# \#}$ & $-0.5183 * * *$ & $-0,0258$ \\
\hline LPC(19:1) & $-0.3856^{*}$ & $-0.7424^{\# \# \#}$ & $-0.6833^{\# \#}$ & $-0.4568 * *$ & -0.1568 \\
\hline LPE(18:2) & $-0.3354 *$ & $-0.7420^{\# \# \#}$ & $-0.6329^{\#}$ & $-0.5196^{* * *}$ & -0.1478 \\
\hline Glycerophosphocholine & -0.1958 & $-0.7419^{\# \# \#}$ & $-0.6717^{\# \#}$ & $-0.3537 *$ & -0.2752 \\
\hline PC(21:1) & $-0.3677 *$ & -0.7416 & $-0.6801^{\# \#}$ & $-0.5042 * * *$ & -0.1872 \\
\hline LPE(22:1) & $-0.4061^{* *}$ & $-0.7368^{\# \# \#}$ & $-0.7082^{\# \# \#}$ & $-0.4431 * *$ & -0.2346 \\
\hline LPI(22:6) & $-0.4143 *$ & $-0.7279^{\# \# \#}$ & $-0.6426^{\# \#}$ & $-0.4641 * *$ & -0.0894 \\
\hline $\operatorname{LPA}(18: 2)$ & $-0.3502 *$ & $-0.7241^{\# \# \#}$ & $-0.6421^{\#}$ & $-0.4497 * *$ & -0.1840 \\
\hline LPI(16:1) & $-0.4845 * *$ & $-0.7206^{\# \#}$ & $-0.6073 * * *$ & $-0.4985 * *$ & -0.0478 \\
\hline $\begin{array}{l}\text { Docosahexaenoic acid } \\
\text { methylester }\end{array}$ & $-0.3914 *$ & $-0.7186^{\# \# \#}$ & $-0.6787^{\# \#}$ & $-0.4716^{* *}$ & -0.2203 \\
\hline LPC(O-18:0)sn-1 & $-0.3112 *$ & $-0.7150^{\# \# \#}$ & $-0.6155^{\#}$ & $-0.4048 * *$ & $-0.3264 *$ \\
\hline LPE(22:6) & $-0.3774 *$ & $-0.7080^{\# \#}$ & $-0.6403^{\# \#}$ & $-0.4790 * *$ & -0.1102 \\
\hline $\begin{array}{l}\text { Dihydroxy-cholestanoyl } \\
\text { taurine }\end{array}$ & -0.2415 & $-0.7065^{\# \# \#}$ & $-0.6010^{\#}$ & $-0.3503 *$ & -0.0955 \\
\hline LPC(22:5)sn-2 & $-0.3581 *$ & $-0.6956^{\# \#}$ & $-0.5983^{\#}$ & $-0.4273^{* *}$ & $-0,1983$ \\
\hline LPC(22:4)sn-2 & $-0.3917 *$ & $-0.6953^{\# \#}$ & $-0.6136^{\#}$ & $-0.4745^{* *}$ & -0.1547 \\
\hline LPC(20:2) & $-0.3725 *$ & $-0.6952^{\# \#}$ & $-0.5867^{\#}$ & $-0.4072 * *$ & -0.2029 \\
\hline LPC(22:6)sn-2 & $-0.3288 *$ & $-0.6915^{\# \#}$ & $-0.5956 * * *$ & $-0.4266^{* *}$ & -0.1448 \\
\hline LPC(O-16:0)sn-1 & -0.2589 & $-0.6899^{\# \#}$ & $-0.5984^{\#}$ & -0.3703 & -0.2656 \\
\hline LPC(20:1)sn-1 & $-0.3770 *$ & $-0.6896^{\# \#}$ & $-0.6290^{\#}$ & $-0.4484 * *$ & $-0,3447 *$ \\
\hline PC(O-38:6) or PC(P-38:5) & $-0.3214^{*}$ & $-0.6867^{\# \#}$ & $-0.5977 * * *$ & -0.3761 & -0.1471 \\
\hline $\begin{array}{l}\text { Epoxy-dimethyl- } \\
\text { cyclocholestanol }\end{array}$ & -0.2428 & $-0.6857^{\# \#}$ & $-0.6273^{\#}$ & $-0.3922 *$ & -0.0507 \\
\hline LPE(O-18:1) or LPE(P-18:0) & -0.2607 & $-0.6843^{\# \#}$ & $-0.6250^{\#}$ & $-0.3637^{*}$ & -0.1600 \\
\hline LPC(O-18:1) or LPC (P-18:0) & $-0.3295^{*}$ & $-0.6823^{\# \#}$ & $-0.6303^{\#}$ & $-0.3168^{*}$ & $-0.3789^{*}$ \\
\hline LPI(20:3) & $-0.5047^{* *}$ & $-0.6811^{\# \#}$ & $-0.6662^{\# \#}$ & $-0.5162 * *$ & -0.2676 \\
\hline PE(36:3) & -0.3061 & $-0.6810^{\# \#}$ & $-0.6929^{\# \#}$ & -0.2945 & $-0.3165^{*}$ \\
\hline LPE(P-16:0) & -0.1444 & $-0.6781^{\# \#}$ & $-0.5392 * * *$ & -0.2141 & -0.2523 \\
\hline LPE(20:0) & -0.3108 & $-0.6712^{\# \#}$ & $-0.6208^{\#}$ & $-0.4380^{* *}$ & -0.1533 \\
\hline LPE(15:1) & $-0,2123$ & $-0.6670^{\# \#}$ & $-0.6027^{\#}$ & $-0.3163 *$ & -0.1365 \\
\hline Palmitoyl isopropylamine & -0.2839 & $-0.6634^{\# \#}$ & $-0.5471 * * *$ & $-0.3960 *$ & -0.1194 \\
\hline
\end{tabular}




\begin{tabular}{|l|c|c|c|c|c|}
\hline Oleoyl ethylamine & $-0.3307^{*}$ & $-0.6629^{\# \#}$ & $-0.5277^{* * *}$ & $-0.4320^{* *}$ & -0.1164 \\
\hline PC(19:0) & -0.2964 & $-0.6592^{\# \#}$ & $-0.6035^{* * *}$ & $-0.3338^{*}$ & -0.2048 \\
\hline LPC(18:0) & -0.2370 & $-0.6582^{\# \#}$ & $-0.5625^{* * *}$ & $-0.3575^{*}$ & -0.2002 \\
\hline Lipoxin C4 & $-0.3367^{*}$ & $-0.6577^{\# \#}$ & $-0.6010^{\#}$ & $-0.4673^{* *}$ & -0.1615 \\
\hline
\end{tabular}

Only metabolites with Spearman coefficients $\left(\mathrm{r}_{s}\right)$ higher than 0.65 for correlation with $2 \mathrm{~h}$ glucose are shown. * $P<0.05$; ** $P<0.01$; *** $P<0.001$; \# $P<0.0001$; \#\# $P<0.00001$; \#\#\# $P<$ 0.000001 . Correlations with $P$ value $<0.0001$ are color in gray. 
Table 4. ROC analysis.

\begin{tabular}{|c|c|c|c|c|c|}
\hline Compound & $\mathbf{A U C}$ & $P$ value & $\begin{array}{c}\text { Sensitivity } \\
(\%)\end{array}$ & $\begin{array}{c}\text { Specificity } \\
(\%)\end{array}$ & $\begin{array}{l}\text { Likelihood } \\
\text { Ratio }\end{array}$ \\
\hline LPC(18:1) & 0.988 & $<0.0001$ & 100 & 95 & 19 \\
\hline LPC(18:2) & 0.990 & $<0.0001$ & 100 & 95 & 20 \\
\hline LPC(19:1) & 0.960 & $<0.0001$ & 90 & 95 & 18 \\
\hline LPC(20:1) & 0.940 & $<0.0001$ & 90 & 95 & 18 \\
\hline LPC(20:2) & 0.980 & $<0.0001$ & 95 & 95 & 19 \\
\hline LPC(20:4) & 0.980 & $<0.0001$ & 95 & 95 & 19 \\
\hline LPC(20:5) & 0.988 & $<0.0001$ & 100 & 95 & 20 \\
\hline LPC(22:4) & 0.983 & $<0.0001$ & 95 & 95 & 19 \\
\hline LPC(22:5) & 0.990 & $<0.0001$ & 90 & 95 & 18 \\
\hline LPC(22:6) & 0.971 & $<0.0001$ & 90 & 95 & 18 \\
\hline LPE(18:2) & 0.965 & $<0.0001$ & 80 & 95 & 16 \\
\hline LPE(20:0) & 0,950 & $<0.0001$ & 85 & 95 & 17 \\
\hline LPE(20:1) & 0.995 & $<0.0001$ & 100 & 95 & 20 \\
\hline LPE(20:2) & 0.995 & $<0.0001$ & 100 & 95 & 20 \\
\hline LPE(22:1) & 0.945 & $<0.0001$ & 95 & 95 & 19 \\
\hline LPE(22:4) & 0.985 & $<0.0001$ & 100 & 95 & 20 \\
\hline LPE(22:6) & 0.958 & $<0.0001$ & 75 & 95 & 15 \\
\hline LPS(20:0) & 0.995 & $<0.0001$ & 100 & 95 & 20 \\
\hline LPS(21:0) & 0.995 & $<0.0001$ & 100 & 95 & 20 \\
\hline LPS(22:0) & 0.960 & $<0.0001$ & 90 & 95 & 18 \\
\hline LPI(18:2) & 0.990 & $<0.0001$ & 100 & 95 & 20 \\
\hline LPI(20:3) & 0.969 & $<0.0001$ & 75 & 95 & 15 \\
\hline LPI(20:4) & 0.980 & $<0.0001$ & 100 & 95 & 19 \\
\hline LPI(22:6) & 0,973 & $<0.0001$ & 95 & 95 & 19 \\
\hline LPA(18:2) & 0.990 & $<0.0001$ & 100 & 95 & 20 \\
\hline $\mathrm{PC}(21: 1)$ & 0.988 & $<0.0001$ & 100 & 95 & 20 \\
\hline $\begin{array}{l}\text { Docosahexaenoic acid } \\
\text { methylester }\end{array}$ & 0.975 & $<0.0001$ & 95 & 95 & 19 \\
\hline Araquidonate methylester & 0.968 & $<0.0001$ & 95 & 95 & 19 \\
\hline Glycerophosphocholine & 0.959 & $<0.0001$ & 94 & 95 & 19 \\
\hline Lipoxin C4 & 0.943 & $<0.0001$ & 90 & 95 & 18 \\
\hline $\begin{array}{l}\text { Trihydroxy-cholestanoyl } \\
\text { taurine }\end{array}$ & 0.995 & $<0.0001$ & 100 & 95 & 20 \\
\hline $\begin{array}{l}\text { Taurolithocholic } \\
\text { glucuronide }\end{array}$ & 0.990 & $<0.0001$ & 95 & 95 & 19 \\
\hline
\end{tabular}

Receiver-operating characteristic (ROC) curves were prepared by plotting the sensitivity against the corresponding false-positive rate (100-specificity). Table shows the area under the curve (AUC), and the best sensitivity, specificity and likelyhood ratio for a selected cut-off of each parameter. 


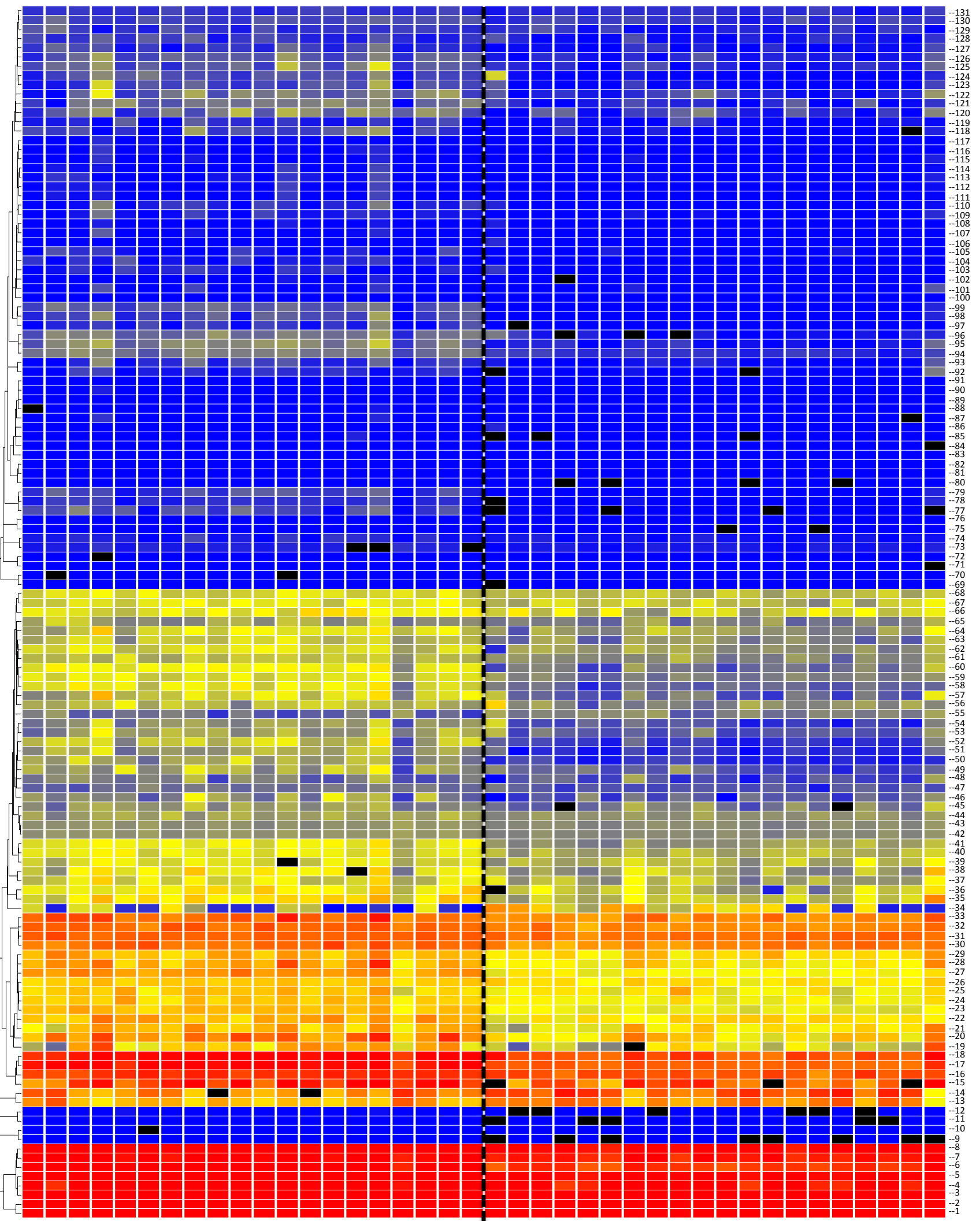

$$
\text { ᄂ }
$$

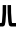


Control

A

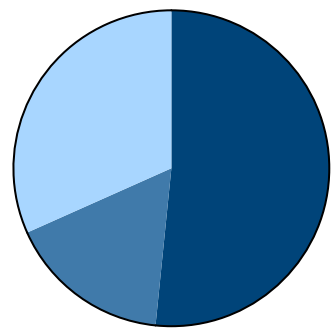

LPC Total $=2.655 \mathrm{E}+8$

Control

B

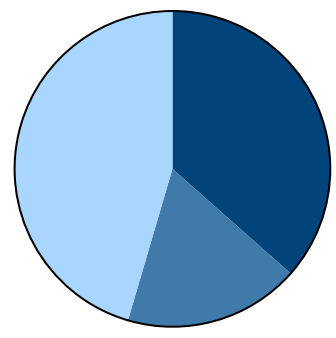

LPE Total $=3.696 \mathrm{E}+7$

Control

C

LPI Total=4.409E+6

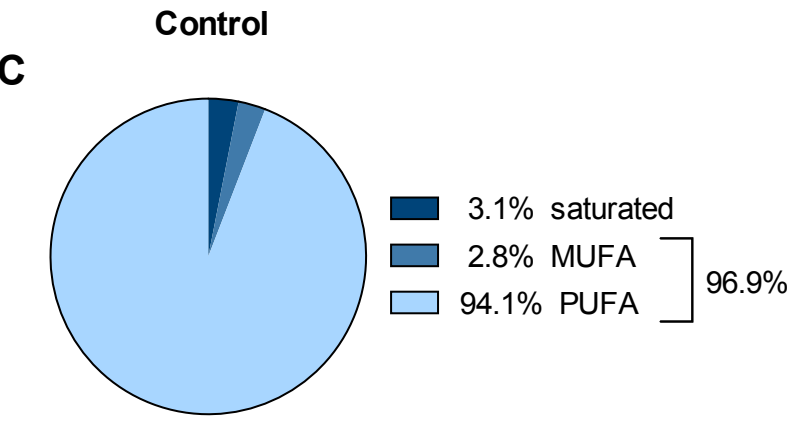

$51.6 \%$ saturated

$16.7 \%$ MUFA

$31.7 \%$ PUFA

$36.5 \%$ saturated

$18.0 \%$ MUFA

45.5\% PUFA _

$63.5 \%$

$$
45.5 \% \text { PUFA }
$$

$3.1 \%$ saturated

$2.8 \%$ MUFA

94.1\% PUFA

$96.9 \%$

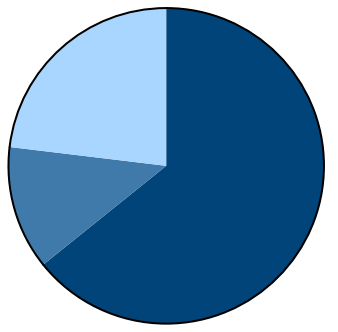

LPC Total $=1.325 \mathrm{E}+8$

GDM

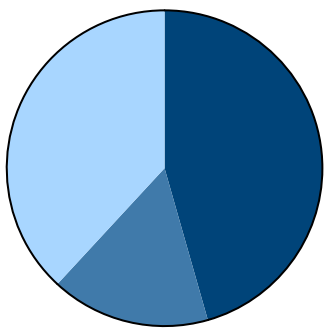

LPE Total=1.797E+7

GDM

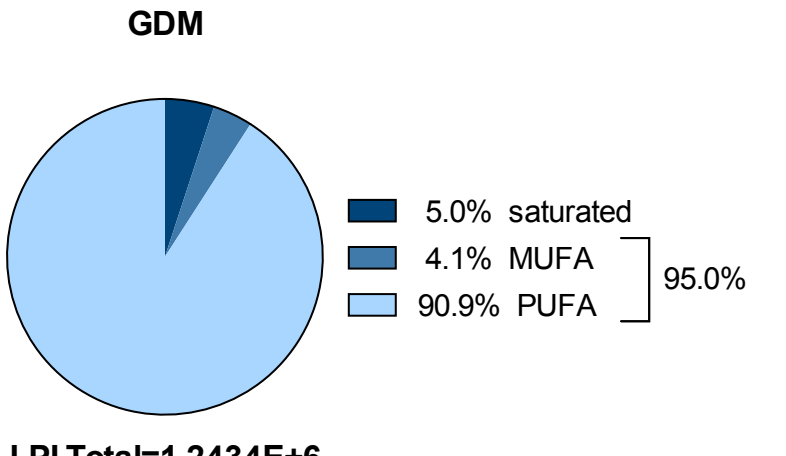

LPI Total=1.2434E+6
$64.2 \%$ saturated

$12.7 \%$ MUFA

$23.1 \%$ PUFA

$45.6 \%$ saturated

$16.3 \%$ MUFA

$38.1 \%$ PUFA 


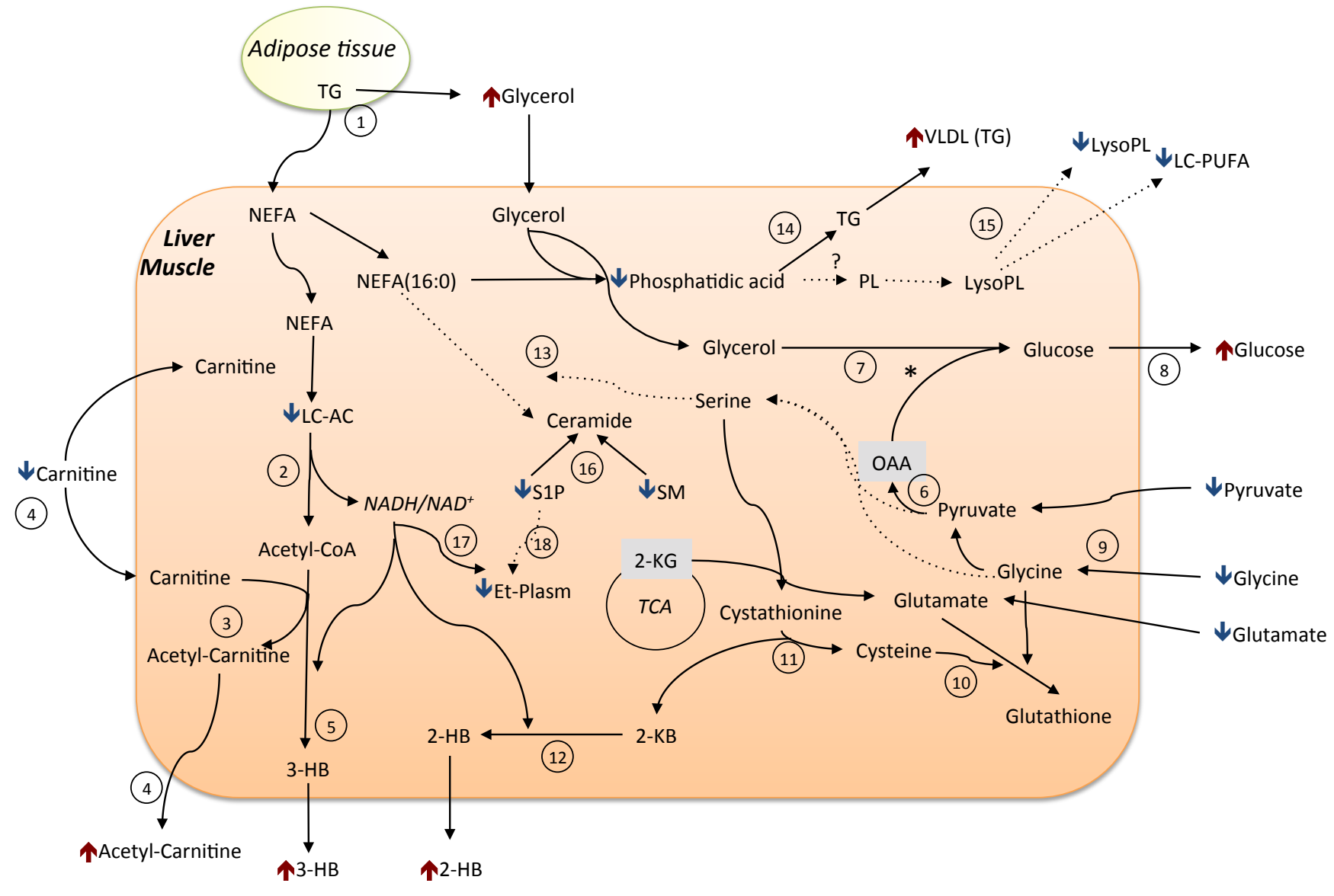

